NBER WORKING PAPER SERIES

\title{
SATISFICING CONTRACTS
}

\author{
Patrick Bolton \\ Antoine Faure-Grimaud \\ Working Paper 14654 \\ http://www.nber.org/papers/w14654
}

\author{
NATIONAL BUREAU OF ECONOMIC RESEARCH \\ 1050 Massachusetts Avenue \\ Cambridge, MA 02138 \\ January 2009
}

We owe special thanks to Denis Gromb for detailed comments. We also thank Mathias Dewatripont, Leonardo Felli, Ronald Gilson, Oliver Hart, Bengt Holmström, Bentley MacLeod, Eric Maskin, John Moore, Michele Piccione, Alan Schwartz, Andy Skrzypacz, Jean Tirole and seminar participants at the London School of Economics, Bocconi University, the University of Cambridge, the Studienzentrum Gerzensee, the Utah Winter Business Economics Conference, the London Business School Conference on Contracts and Bounded Rationality, and the Columbia Law School Conference on Business Law and Innovation for their comments. The views expressed herein are those of the author(s) and do not necessarily reflect the views of the National Bureau of Economic Research.

NBER working papers are circulated for discussion and comment purposes. They have not been peerreviewed or been subject to the review by the NBER Board of Directors that accompanies official NBER publications.

(C) 2009 by Patrick Bolton and Antoine Faure-Grimaud. All rights reserved. Short sections of text, not to exceed two paragraphs, may be quoted without explicit permission provided that full credit, including $\odot$ notice, is given to the source. 
Satisficing Contracts

Patrick Bolton and Antoine Faure-Grimaud

NBER Working Paper No. 14654

January 2009

JEL No. C61,D81,D84,D86

\begin{abstract}
$\underline{\text { ABSTRACT }}$
We propose a model of equilibrium contracting between two agents who are "boundedly rational" in the sense that they face time-costs of deliberating current and future transactions. We show that equilibrium contracts may be incomplete and assign control rights: they may leave some enforceable future transactions unspecified and instead specify which agent has the right to decide these transactions. Control rights allow the controlling agent to defer time-consuming deliberations on those transactions to a later date, making her less inclined to prolong negotiations over an initial incomplete contract. Still, agents tend to resolve conflicts up-front by writing more complete initial contracts. A more complete contract can take the form of either a finer adaptation to future contingencies, or greater coarseness. Either way, conflicts among contracting agents tend to result in excessively complete contracts in the sense that the maximization of joint payoffs would result in less up-front deliberation.
\end{abstract}

Patrick Bolton

Columbia Business School

804 Uris Hall

New York, NY 10027

and NBER

pb2208@columbia.edu

Antoine Faure-Grimaud

Financial Markets Group

London School of Economics

A.Faure-Grimaud@1se.ac.uk 


\section{Introduction}

This paper analyzes a contracting model with two agents, each facing thinking costs, in which equilibrium incomplete contracts arise endogenously. The basic situation we model is an investment in a partnership or an ongoing new venture. The contract the agents write specifies in a more or less complete manner what action-plan they agree to undertake initially, and how the proceeds from the venture are to be shared. In any given state of nature both agents face costs in thinking through optimal decisions in that state. Therefore an optimal contract that maximizes gains from trade net of thinking costs is generally incomplete in the sense that it is not based on all the information potentially available to agents in all states of nature. By introducing positive thinking or deliberation costs into an otherwise standard contracting framework, it is thus possible to formulate a theory of endogenously incomplete contracts.

As Oliver Hart and others have observed, to understand why contracts are incomplete and what determines the degree of incompleteness of contracts one ultimately needs to appeal to the contracting agents' bounded rationality:

"In reality, a great deal of contractual incompleteness is undoubtedly linked to the inability of agents not only to contract very carefully about the future, but also to think very carefully about the utility consequences of their actions. It would therefore be highly desirable to relax the assumption that agents are unboundedly rational." [Hart, 1995, p. 81]

The only departure from full rationality we explore in this article is time-costs in thinking through optimal transactions. ${ }^{1}$ As will become clear in the formal analysis below, even such a minimal departure introduces major new conceptual issues. But in spite of these complications our quasi-rational model captures several important features of incomplete contracting observed in practice.

One first basic result is that boundedly rational agents write what we call satisficing contracts, which do not fully exploit all gains from trade that would be available to agents

\footnotetext{
${ }^{1}$ We develop the model of decision-making with positive deliberation costs more fully in Bolton and FaureGrimaud (2008). Our model builds on earlier work on decision-making with deliberation costs by Simon (1955) and Conlisk $(1980,1988,1996)$ among others, and on the literature on multiarmed bandits by Gittins and Jones (1974), Rothschild (1974), Gittins (1979), Berry and Frystedt (1985) and Whittle (1980, 1982).
} 
who face no deliberation costs. ${ }^{2}$ In equilibrium, agents don't waste time resolving all future transactions and instead leave many decisions to be determined later. Agents will tend to settle on more incomplete action-plans when they have broadly aligned interests, and when they all expect to benefit substantially from the deal. Note, in particular, that boundedly rational agents choose to leave transactions unresolved in perfectly foreseeable, describable and enforceable contingencies, if these contingencies are sufficiently unlikely or distant, or if they don't affect expected payoffs much. In addition, contracts become more and more detailed over time, as agents complete the contract in light of new information.

We refer to such contracts as incomplete contracts to the extent that they do not involve complete ex-ante information acquisition on payoffs of all transactions in all states, and they do not just specify state-contingent transactions based only on the information agents have acquired ex ante. Contracts can always be made contingent on all the information available to the contracting parties and in that sense contracts can always be complete. That said, when agents choose to defer information acquisition on certain transactions to when a given state of nature arises, they may as well write what is more commonly referred to as an incomplete contract, namely a contract where the ultimate transaction to be undertaken in that state is left unspecified and where a controlling agent has the right to determine the transaction should that state of nature arise (see Grossman and Hart, 1986, and Hart and Moore, 1988). Such an incomplete contract would often yield the same expected payoff as an optimal contract that is based on all the information agents choose to acquire in a particular state, and would be a lot simpler to write.

The main results from our analysis are as follows: First, incomplete contracts specifying control rights may emerge in equilibrium (when such contracts are not strictly dominated by a complete contract with the same equilibrium information acquisition). The rationale for control rights in our model - defined as rights to decide between different transactions in contingencies left out of the initial contract - is that the holder of these rights benefits by having the option to defer thinking about future decisions. Second, control rights tend to be allocated to the more cautious party. Indeed, the more cautious party is then more willing to close the deal quickly, even though it has not had the time to think through all contingencies, in the knowledge that thanks to its control rights it can impose its most favored decision in

\footnotetext{
${ }^{2}$ We borrow Simon's notion of satisficing for decision problems of boundedly rational agents to describe a contracting problem between such agents (see Simon, 1955, Radner, 1975, and Radner and Rothschild, 1975). Interestingly, although satisficing behavior has been explored extensively in decision problems it has not, to our knowledge, been extended to a contracting problem.
} 
the unexplored contingencies.

Third, the sharp distinction between a first contract negotiation phase followed by a phase of execution of the contract usually made in the contract theory literature is no longer justified in our setup. Contracts are completed over time and negotiations about aspects that have been left out initially can be ongoing. In particular, the contracting agents may choose to begin negotiations by writing a preliminary contract specifying the broad outlines of a deal and committing the agents to the deal. The agents then continue with a further exploration phase (which may be thought of as a form of due diligence) before deciding whether to go ahead with the venture and agreeing to a detailed contract. Interestingly, a party with all the bargaining power may choose to leave rents to the other party, so as to meet its prior aspiration level - that is, the level before it has had time to think through all contingencies - and thus persuade it to sign on more quickly.

Fourth, when agents' objectives conflict more, equilibrium contracts are more complete. The main reason is that each agent may be concerned about the detrimental exercise of control by the other agent, so that abuse of power cannot be limited by just allocating control to the agent that is least likely to abuse power. In such situations the exercise of control may have to be circumscribed contractually by writing more complete contracts. Another reason is that when agents have conflicting goals they are less willing to truthfully share their thoughts, so that the net benefit of leaving transactions to be fine-tuned later is reduced.

This analysis thus provides new foundations for incomplete contracts and the role of control rights. In our model equilibrium contracts may be incomplete even though more complete contracts (relying on more information acquisition) are enforceable. Similarly, contractual completeness increases over time even though enforceability remains unchanged. This is in our view a critical difference with first generation models of incomplete contracts. Two important implications immediately follow. First, our framework allows for contractual innovation by the contracting agents independently of any changes in legal enforcement. Second, changes in legal enforcement may have no effect on equilibrium contracts if enforcement constraints were not binding in the first place.

There can be substantial contractual innovation unprompted by changes in legal enforcement as, Kaplan, Martel, and Stromberg (2007) have strikingly documented. In their study they track the evolution of venture capital (VC) contracts in over twenty countries outside the U.S. and compare them to U.S. VC contracts. A key finding is that although contracts 
differ across jurisdictions, and thus seem at first sight to be constrained by local legal enforcement, the more experienced VCs end up writing the same U.S.-style contracts independently of the local legal environment. Bienz and Walz (2008) provide other empirical support and find that exit rights for VCs are generally only written into the contract at later financing rounds, consistent with our hypothesis that VCs focus on exit rights only once exit issues become more pressing. They also find that older, hence more experienced, VCs write more complete contracts by including more control rights clauses into contracts. Another common VC contracting practice they highlight is the use of "term sheets", a form of preliminary contract containing general clauses of the form "other terms and conditions customary to venture capital financing will apply".

In the first generation models of incomplete contracting theories à la Grossman and Hart (1986) and Hart and Moore (1988) agents are fully rational but unable to contractually specify transactions in some states of nature due to exogenous verifiability or describability constraints. Being fully rational, agents will always write the most complete contract they can, and contractual efficiency is always constrained by enforcement effectiveness. Moreover, since contract incompleteness is entirely driven by exogenous enforcement constraints, the contracting agents are unable to limit discretion contractually and are reduced to only determining optimal control allocations over decisions that cannot be written into the contract. Except that, as Maskin and Tirole (1999) have observed, rational agents may actually be able to write complete contracts by circumventing enforcement constraints through sophisticated Maskin (revelation) schemes.

Our analysis is closely related to a second generation of incomplete contracting theories, which includes Anderlini and Felli (1994, 1999, 2001), Al Najjar, Anderlini and Felli (2002), MacLeod (2000), Battigalli and Maggi (2002), Bajari and Tadelis (2001), and Hart and Moore (2007). ${ }^{3}$ These studies also provide theories of endogenous contractual incompleteness, but based on other transaction costs, such as costs of writing detailed contracts, or limits on language in describing certain transactions or contingencies. In closely related independent work, Tirole (2008) also considers contracting between two boundedly rational agents. Contracts in his set-up always specify a given action to be taken, but they are less likely to be renegotiated (more complete) when contracting agents have incurred larger cognitive costs. Although the basic setup he considers is quite different from ours, similar themes and results emerge, such as the endogenous incompleteness of contracts and the ex-

\footnotetext{
${ }^{3}$ See also the earlier theory of Dye (1985).
} 
cessive completeness of equilibrium contracts. Unlike in our model, Tirole only allows for effort costs of cognition and does not explore the dynamics of contractual completion. He focuses on a holdup problem and the value of thinking in his model comes from the greater likelihood of solving a hold-up problem contractually up-front. Incurring thinking costs is valuable primarily to the agent making sunk investments and is otherwise of no social value. This is the main reason why contracts tend to be excessively complete.

Finally, our model and the second generation theories can be seen as attempts to formalize different aspects of Williamson's $(1979,1985)$ transactions costs theory. As Williamson has forcefully argued, contracts in reality are likely to be incomplete primarily due to the costs of specifying transactions on paper and due to the bounded rationality of contracting agents. Interestingly, a major theme in Williamson's theory is that a key role of organizations is to move enforcement away from courts and inside firms, thereby dampening potential conflicts between agents and thus increasing the efficiency of incomplete contracts. As we suggest in the conclusion, it may also be beneficial in our framework to impose limits on the enforcement of contracts that allow the controlling party to exercise authority in an abusive way.

The paper proceeds as follows. Section 2 presents our model of contracting between two boundedly rational agents. Section 3 characterizes satisficing contracts under the assumptions of non-transferable utility and communication of hard information. Section 4 considers extensions to communication of soft information and transferable utility. Section 5 concludes and an appendix contains the more involved proofs.

\section{The Model}

Two infinitely-lived agents, $A$ and $B$, can join forces to undertake a new venture at time $t=0$. The venture requires initial funding $I>0$ from each agent. If investments are sunk at date $t \geq 0$ then at date $t+1$ the venture ends up in one of two equally likely states: $\theta \in\left\{\theta_{1}, \theta_{2}\right\}$. In state $\theta_{1}$ the two agents get the same known payoff $\pi \geq 0$. In state $\theta_{2}$, the two agents face the collective decision of choosing between a safe and a risky action. The safe action yields known payoffs $S_{A}$ and $S_{B}$, while the risky action yields either $\left(\underline{R}_{A}, \underline{R}_{B}\right)$ or $\left(\bar{R}_{A}, \bar{R}_{B}\right)$. To make the problem non-trivial we assume:

$$
\bar{R} \equiv \bar{R}_{A}+\bar{R}_{B}>S \equiv S_{A}+S_{B}>\underline{R} \equiv \underline{R}_{A}+\underline{R}_{B}
$$


Thus the only uncertainty in the model is which state of nature will occur and the payoff of the risky action in state $\theta_{2}$.

At $t=0$, the beginning of the game, neither agent $k=A, B$ knows the true value of $R_{k}$ and each agent starts out with prior belief $\nu=\operatorname{Pr}(R=\bar{R})$, which can be revised by engaging in thought-experimentation over time as follows. If agent $k$ experiments in a given period he privately observes $R_{k}$ with probability $\lambda_{k}$ and nothing otherwise.

As long as neither agent has found out the true payoff of the risky action, either agent can and may want to continue to engage in thought experimentation. The agents can engage in thought experimentation before or after signing a contract, and before or after the state of nature $\theta$ is realized. Both agents discount future returns by the same factor $\delta \leq 1$.

\section{Timing of the Game:}

We shall make the following timing assumptions:

\section{Technological Timing}

At date 0 , the agents can invest $I$ right away or postpone investment. They can also engage in one round of thought experimentation. Investment can only be undertaken if both agents agree to invest.

Subsequent periods are essentially identical to date 0 until investment takes place. The only difference is that the agents may have been able to update their beliefs about the payoff of the risky action in state $\theta_{2}$. Once investment has been completed, either state of nature $\theta_{1}$ or $\theta_{2}$ is realized one period later. When state $\theta_{2}$ occurs the agents either engage in more thinking, or choose an action. Once an action has been chosen, payoffs are realized and the game ends.

\section{Timing of the Negotiation Game}

For expositional convenience we divide each period into two stages: a first stage when a contract (or renegotiation) offer is made and possibly accepted, and a second stage as described in the technological timing above.

We make the extreme simplifying assumption that at the beginning of date 0 nature randomly gives one of the two agents (the proposer) all the bargaining power and the exclusive right to make all contract offers. In each period until the contract is signed the proposer can choose to wait or offer a contract to the other party (the receiver), who can either accept or reject the offer. If no offer is made or if the offer is rejected, the game moves to the next period and starts over again. If the offer is accepted the agents move on with the 
venture. If the contract is complete, post-contractual play is fully specified. If the contract is incomplete, the agents play a post-contractual game, which we describe in detail below.

\section{Information and Contracts}

We assume that at date 0 neither agent has any private information about $R_{k}$ and that agents' prior belief $\nu$ is common knowledge. In subsequent periods, however, each agent can obtain private information about their payoffs through thought-experimentation. Each agent can elect to disclose some of that information to the other agent. We shall distinguish between the cases of hard information, in which information can be credibly disclosed, and soft information, where communication is cheap talk.

We also distinguish between two polar contracting environments: one where the agents' utility is perfectly transferable (the $T U$ case) and the other where utility is non-transferable (the NTU case).

Throughout most of our analysis we focus on the case where utility is non-transferable and where private information can be credibly disclosed. We consider the TU case and cheap talk communication in an extension.

\section{Assumptions on Payoffs}

We denote by

$$
\rho_{k}^{*} \equiv \nu \max \left\{\bar{R}_{k}, S_{k}\right\}+(1-\nu) \max \left\{\underline{R}_{k}, S_{k}\right\}
$$

each party's expected payoff under their preferred ex-post action choice and by

$$
\rho_{k} \equiv \nu \bar{R}_{k}+(1-\nu) \underline{R}_{k}
$$

the expected payoff of the risky action. We make the following assumptions on payoffs throughout the analysis:

$$
\text { Assumption A1: } \frac{\delta\left(\pi+S_{k}\right)}{2}>I \text { and } \rho_{k}>S_{k} \text {. }
$$

Assumption A1 guarantees that the project is valuable for both agents when the safe action is chosen in state $\theta_{2}$. Moreover, both agents prefer the risky over the safe action given their prior beliefs. As will become clear below, this assumption is not essential and our analysis can be extended to the case where agents prefer the safe over the risky action when they are uninformed.

We consider in turn the situations where the agents have congruent underlying objectives over which action to choose, and where they have conflicting objectives on the preferred 
action-plan. In the first situation the two agents can only disagree on how quickly to act, or, in other words, on how far ahead to plan.

\section{Satisficing Contracts under Congruent Objectives}

In this section we consider the contracting game when the two agents' objectives are congruent. We define the agents' underlying preferences to be congruent when the following assumption holds:

Assumption A2: $\bar{R}_{A}>S_{A}>\underline{R}_{A}$ and $\bar{R}_{B}>S_{B}>\underline{R}_{B}$.

Under assumption A2 both agents agree on the action choice once they know the true payoffs.

The contracting game begins at date 0 with nature selecting the contract proposer. We shall take it that agent $A$ is the proposer and $B$ the receiver. If $B$ accepts $A$ 's offer, the continuation game is dictated by the terms of the contract. If $A$ does not make an offer or if $B$ rejects the contract, then each agent engages in one round of thought experimentation and communication before moving on to the next period. In the next period again $A$ gets to make a contract offer, and so on, until an offer is accepted by $B$.

The set of relevant contract offers for $A$ under our assumption that utility is not transferable can be reduced to essentially five contracts $^{4}, \mathbb{C}=\left\{C_{R}, C_{S}, C_{A}, C_{B}, C_{A B}\right\}$, and any probability distribution over $\mathbb{C}$, where:

1. $C_{R}$ requires the immediate choice of the risky action $r$ in state $\theta_{2}$ following investment;

2. $C_{S}$ requires the immediate choice of the safe action $s$ in state $\theta_{2}$ following investment;

3. $C_{A}$ allocates all control rights to agent $A$ following investment. The controlling party can decide which action to take in state $\theta_{2}$ at any time she wants;

4. $C_{B}$ is identical to $C_{A}$ except that it allocates all control rights to agent $B$;

5. $C_{A B}$ is identical to $C_{A}$ except that it requires unanimous agreement to select an action;

\footnotetext{
${ }^{4}$ There may also be a sixth contract, which we refer to as a preliminary contract. Under this contract, which we denote by $C_{\alpha}$, the parties agree to first find out what the payoffs of the risky action are and to invest only once they have agreed on a final contract $C \in\left\{C_{R}, C_{S}\right\}$. We consider this contract in subsection 4.2 .
} 
Even if agents have congruent underlying preferences they may still have disagreements under incomplete information. In particular, they may have different preferences on how quickly to invest due to differences in how quickly they are able to think. To see this, note first that when the agents engage in thought-experimentation in a given period, and share their thoughts, they uncover the true payoff of the risky action in a given period of time with probability:

$$
\Lambda \equiv 1-\left(1-\lambda_{A}\right)\left(1-\lambda_{B}\right)
$$

Now, suppose that the agents find themselves in state $\theta_{2}$ and are uninformed. If the two agents delay any action choice and engage in thought-experimentation until they learn the true payoff $R_{k}$ they can each expect to get:

$$
\Lambda \rho_{k}^{*}+\Lambda(1-\Lambda) \delta \rho_{k}^{*}+\Lambda(1-\Lambda)^{2} \delta^{2} \rho_{k}^{*}+\ldots=\widehat{\Lambda} \rho_{k}^{*}
$$

where

$$
\widehat{\Lambda}=\frac{\Lambda}{1-(1-\Lambda) \delta}
$$

can be interpreted as an effective discount factor.

Clearly, it is possible that:

$$
\widehat{\Lambda} \rho_{A}^{*}<\rho_{A} \text { and } \widehat{\Lambda} \rho_{B}^{*}>\rho_{B}
$$

even under assumption A2. In this case the two agents disagree on the best course of action in state $\theta_{2}: A$ prefers to take the risky action immediately, while $B$ prefers to learn $R_{k}$ first before deciding on an action.

It is helpful to begin our analysis by studying first the contracting outcome when both agents have "unbounded rationality". This corresponds to two different situations in our model: either there is nothing to be learned $(\nu=0$ or $\nu=1)$ or nothing that can be learned $\left(\lambda_{A}=\lambda_{B}=0\right)$.

\subsection{Two 'unbounded rationality' benchmarks}

When either $\nu=1$ or $\nu=0$, the equilibrium outcome of the contracting game is to sign a contract requiring immediate investment. If $\nu=1$ the contract specifies the risky action and if $\nu=0$ it specifies the safe action in state $\theta_{2}$. Indeed, in this case payoffs are known and when $\nu=1$ agents agree that the best action choice in state $\theta_{2}$ is the risky action (as $\bar{R}_{k}>S_{k}$ by assumption A1) and when $\nu=0$ they agree that the safe action is best (as 
$\left.\underline{R}_{k}<S_{k}\right)$. Another optimal contract is give discretion to one or both of agents over the action choice in state $\theta_{2}$. The agents' respective payoffs are:

$$
-I+\frac{\delta \pi}{2}+\frac{\delta}{2} \bar{R}_{k}
$$

when the risky action is optimal, and

$$
-I+\frac{\delta \pi}{2}+\frac{\delta}{2} S_{k}
$$

when the safe action is optimal.

To see that this is an equilibrium outcome note that since $A$ and $B$ 's preferred actionplan is the same, when $A$ offers a contract requiring investment at date 0 and specifying his preferred action-plan, $B$ is strictly better off accepting the offer.

For the same reason, when $\lambda_{A}=\lambda_{B}=0$, the agents sign a contract at date 0 agreeing to invest immediately and to take the risky action in state $\theta_{2}$, since $\rho_{k}>S_{k}$ under assumption A1.

Importantly, in both cases there is no (strict) role for control rights and the initial contract fully specifies the entire action-plan. ${ }^{5}$ This is not surprising given that the two agents can write fully enforceable complete contracts. ${ }^{6}$

In contrast, as we shall show below, boundedly rational agents may agree on an incomplete contract, which leaves open the action choice in state $\theta_{2}$ and gives control to one or both agents.

\subsection{Full Disclosure}

The typical contracting game considered in the literature boils down to a contract offer by the proposer followed by an accept/reject decision by the receiver. ${ }^{7}$ The central new difficulty in our game is that both agents can learn something (privately) about their payoffs between two rounds of offers, so that the negotiation game can evolve into a game of incomplete information even though it starts out as a game of symmetric information. This is, we believe, an inevitable feature of any game of contracting between boundedly rational agents,

\footnotetext{
${ }^{5} \mathrm{~A}$ contract giving full control to the proposer or the receiver may also be an equilibrium contract. However, this contract can never be strictly preferred to the optimal complete contract.

${ }^{6}$ As is well known, when rational agents can write complete, state-contingent, fully enforceable contracts under symmetric information there is no role for control (see, e.g. Hart 1995, or Bolton and Dewatripont, 2005).

${ }^{7}$ If the contract is rejected the game ends and each party gets their reservation utility and if the contract is accepted the game proceeds to the implementation phase of the contract.
} 
who can each learn over time about their payoffs in the contracting relation. It turns out, however, that the negotiation game reduces to a game of complete information under our twin assumptions that: i) any information learned can be credibly disclosed and, ii) that the two agents have congruent underlying preferences.

Lemma 1: Under assumptions $\boldsymbol{A} 1$ and $\boldsymbol{A} 2$, a strategy of revealing all new information to the other party is a subgame-perfect equilibrium strategy of the contracting game for each agent.

Proof: This observation follows immediately from the observations that: i) once the information is shared agents have fully congruent objectives under the stated assumptions; and ii) not revealing what a party has learned can only delay the time at which payoffs are received and cannot result in higher payoffs. As each party gets strictly positive payoffs (under assumption $\boldsymbol{A 1}$ ) it follows that immediate truthful disclosure is a weakly dominant strategy.

\subsection{Complete Satisficing Contracts}

We refer to equilibrium contracts as satisficing contracts to convey the idea that when contracting agents face positive deliberation costs they may agree on contracts in equilibrium that are satisfactory but not optimal from the perspective of rational agents who do not incur any deliberation costs. We begin by observing that when the value of thinking is high and the cost of delaying investment is low then satisficing contracts will be complete.

Formally, a situation with a positive value of information and negative costs of delay is characterized by the following assumption on payoffs.

\section{Assumption A3: $\widehat{\Lambda} \rho_{k}^{*}>\rho_{k}$ and $I>\frac{\delta \pi}{2}$}

The first inequality implies that both agents prefer to find out first which action is optimal before taking an action. Indeed, under full disclosure (Lemma 1), agent $k$ expects to get $\widehat{\Lambda} \rho_{k}^{*}$ when both agents set out to think ahead about which action is optimal, while if they immediately choose the preferred action given their prior belief agent $k$ only gets $\rho_{k}$.

The second inequality implies that thinking ahead is not costly and in particular dominates the strategy of immediately investing and waiting for the realization of $\theta$ before thinking about what to do: under the strategy of thinking ahead of the realization of $\theta$ agent $k$ 
obtains:

$$
\widehat{\Lambda}\left(-I+\frac{\delta \pi}{2}+\frac{\delta \rho_{k}^{*}}{2}\right)
$$

while, under the strategy of investing immediately and thinking about the optimal action after the realization of $\theta_{2}$ he gets:

$$
-I+\frac{\delta \pi}{2}+\frac{\delta}{2} \widehat{\Lambda} \rho_{k}^{*}
$$

The former is preferred to the latter strategy if and only if:

$$
\widehat{\Lambda}\left(-I+\frac{\delta \pi}{2}+\frac{\delta \rho_{k}^{*}}{2}\right)>-I+\frac{\delta \pi}{2}+\frac{\delta}{2} \widehat{\Lambda} \rho_{k}^{*}
$$

or, rearranging,

$$
(1-\widehat{\Lambda})\left(-I+\frac{\delta \pi}{2}\right)<0
$$

Under Assumption A3 this inequality always holds, since $\widehat{\Lambda}<1$.

Both agents agree that in state $\theta_{2}$ they will think before acting. Hence, the only advantage of postponing thinking until after $\theta_{2}$ is realized is to avoid delaying investment should state $\theta_{1}$ occur instead. However, under Assumption A3 the expected value of an investment that ends up in state $\theta_{1}$ is negative.

Thus, thinking ahead of investing, and full disclosure is better for both agents than investing before thinking. This implies that an offer by $A$ of either $C_{A}, C_{B}$ or $C_{A B}$ at date 0 would be dominated by a strategy of waiting and thinking before investing. Similarly, offering to invest immediately at date 0 under contract $C_{R}$ is also dominated. Therefore the following proposition must hold.

Proposition 1: (Complete Satisficing Contracts) Under assumptions A1, $\boldsymbol{A} 2$ and A3 the equilibrium of the contracting game involves thinking ahead of investing followed by the offer of either contracts $C_{R}$ or $C_{S}$.

Proof: This follows immediately from the discussion above. An equilibrium strategy for the proposer is to wait and think until the agents have learned and communicated the optimal action. At that point agent $A$ offers $C_{R}$ if the risky action yields a higher payoff or $C_{S}$ if the safe action is preferred. Given $A$ 's strategy, $B$ 's best response is to think until she learns the optimal action, to disclose it to $A$ if she learns it first, and to accept agent $A$ 's subsequent offer. 


\subsection{Incomplete Satisficing Contracts}

In contrast, when there is a cost of delaying investment one should expect satisficing contracts to sometimes be incomplete. Such situations arise under the following assumption.

Assumption A4: $\widehat{\Lambda} \rho_{k}^{*}>\rho_{k}$ and $I<\frac{\delta \pi}{2}$.

Indeed, delaying investment is costly when $I<\frac{\delta \pi}{2}$. In light of our discussion above, it is easy to see that under this assumption the two agents are better off investing immediately and postponing thinking about the optimal action until after the realization of state $\theta_{2}$.

Proposition 2: Under assumptions $\boldsymbol{A 1}, \boldsymbol{A} 2$ and $\boldsymbol{A} 4$ the equilibrium of the contracting game involves an immediate offer of either contracts $C_{A}, C_{B}$ or $C_{A B}$. Either of these contracts is accepted by agent $B$. Following the realization of state $\theta_{2}$ agents think about the optimal action and the controlling party selects the optimal action once it is identified.

Proof: Under assumption A4 condition (3) is violated and therefore both agents prefer to invest at date 0 and think about the optimal action after the realization of $\theta_{2}$. Thus, an equilibrium strategy for $A$ is to offer at date 0 either contracts $C_{A}, C_{B}$ or $C_{A B}$, and to do the same in future periods should $B$ reject the offer and should the agents remain uninformed. Otherwise, if the agents learn the optimal action, offer either contracts $C_{R}$ or $C_{S}$. Party $B$ 's best response to $A$ 's strategy is to accept $A$ 's offer at date 0 .

Under assumption A4, the venture is so profitable that the agents agree to a contract involving immediate investment so as to bring forward the time when they realize the returns from their investment. As they have congruent underlying preferences they do not care who has control, so that either control allocation $C_{A}, C_{B}$ or $C_{A B}$ can be an equilibrium outcome. However we next show that, even when the two agents have congruent underlying preferences, the indeterminacy over control allocations disappears when the two agents have different preferences over how much thinking to undertake before investing.

\subsection{Conflicts over cautiousness and the allocation of control rights}

We now consider situations in which the two agents disagree on how quickly to act and how much planning to undertake before investing. This is the case when assumption $\mathbf{A 5}$ holds. 
Assumption A5: $\widehat{\Lambda} \rho_{A}^{*}<\rho_{A}, \widehat{\Lambda} \rho_{B}^{*} \geq \rho_{B}$ and $I<\frac{\delta \pi}{2}$

Under this assumption $A$ is impatient and prefers to invest immediately and to choose the risky action in state $\theta_{2}$. Agent $B$, on the other hand, is more cautious and prefers to take its time to think through what is the best action in state $\theta_{2}$. But note also that $B$ is more intelligent or a faster thinker than $A$. This is why $B$ wants to think before acting but not $A$.

We shall show that the equilibrium outcome of the contracting game between the two agents under these circumstances may be for the slower but more impatient party to relinquish control to the more patient party, paradoxically as a way of accelerating the implementation of the project. The intuition is that the more patient party may agree to an earlier implementation of the project if she has control, as control gives her the right to block hasty future decisions and thus allows her to defer thinking about future decisions to the time when they arise. As a result she is prepared to commit to the project sooner.

Proposition 3 Under Assumptions A1, A2, A5 and under condition

$$
-I+\frac{\delta \pi}{2}+\frac{\delta \rho_{B}}{2}<\widehat{\Lambda}\left(-I+\frac{\delta \pi}{2}+\frac{\delta \rho_{B}^{*}}{2}\right)
$$

the equilibrium play is to offer immediately contract $C_{B}$ with probability $y^{*}$ and $C_{R}$ with probability $\left(1-y^{*}\right)$, where $y^{*}$ is given by:

$$
-I+\frac{\delta \pi}{2}+y^{*} \widehat{\Lambda}\left(\frac{\delta \rho_{B}^{*}}{2}\right)+\left(1-y^{*}\right) \frac{\delta \rho_{B}}{2}=\widehat{\Lambda}\left[-I+\frac{\delta \pi}{2}+\frac{\delta \rho_{B}^{*}}{2}\right] .
$$

When the reverse condition holds, agent $A$ immediately offers contract $C_{R}$.

Proof: See the Appendix.

Corollary 1: If the more patient party (agent B) is the proposer then this party optimally retains full control.

Under condition (4) $B$ prefers to reject contract offers $C_{R}, C_{S}$ and $C_{A}$, and to keep thinking ahead until she determines the optimal action in state $\theta_{2}$. But $A$ would prefer to get $B$ to agree to invest immediately. He can only do so if $B$ has some guarantees not to be forced into a hasty decision in state $\theta_{2}$. Therefore he grants $B$ some control rights by offering either contracts $C_{B}$ or $C_{A B}$ with positive probability.

In other words, the impatient proposer prefers to leave the action choice in state $\theta_{2}$ open and give up some control, to get the patient agent to commit to the project sooner and 
refrain from spending too much time fine tuning the details of the deal. By giving up control $A$ gives $B$ the option to fine tune details later if needed, and thus avoids the opportunity cost of delaying investment. ${ }^{8} 9$

Propositions 2 and 3 are among our main results. Each proposition establishes first that in a world where complete contracts are fully enforceable, "boundedly rational" agents optimally choose to write incomplete contracts with control rights over future decisions left unspecified in the contract. As in other models of incomplete contracts, Proposition 3 also establishes that the way control is allocated is in part driven by the agents relative bargaining strengths. Thus, the proposer tends to appropriate more control other things equal. But, remarkably, Proposition 3 further establishes that an impatient proposer may choose to give some control rights to the other more patient party, as a way of accelerating the closure of contract negotiations. ${ }^{10}$

There may also be more extreme situations where $A$ has no choice but to wait until $B$ has determined a complete action-plan. We describe a contracting situation below where party $A$ is keen to close a deal immediately but $B$ prefers to think ahead, and where equilibrium play is such that $A$ completely caves in on $B$ 's demands and ends up writing an excessively complete contract (from $A$ 's perspective). This situation differs from the one we have considered in the following way.

Assumption A6: a) $\widehat{\Lambda}\left(-I+\frac{\delta \pi}{2}+\frac{\delta \rho_{A}^{*}}{2}\right)<-I+\frac{\delta \pi}{2}+\frac{\delta \rho_{A}}{2}$,

b) $\widehat{\Lambda} \rho_{B}^{*} \geq \rho_{B}$, and

c) $I>\frac{\delta \pi}{2}$

${ }^{8}$ Note that when Assumption A1 is not satisfied we may have situations where:

$$
\nu \bar{R}_{A}+(1-\nu) \underline{R}_{A}>S_{A} \text { but } \nu \bar{R}_{B}+(1-\nu) \underline{R}_{B}<S_{B}
$$

That is, the two agents have non-congruent objectives ex ante, even though their underlying preferences are congruent. In such situations the logic of Proposition 3 still applies.

\footnotetext{
${ }^{9}$ In situations where the transfer of control rights may not be legally enforceable, party $B$ can still impose her preferred action. However, the optimal contract would then specify a complete action-plan ex ante. Thus, our analysis suggests, paradoxically, that a condition for the emergence of equilibrium incomplete contracts is that the legal infrastructure is sufficiently developed that the enforcement of control rights is possible.

${ }^{10}$ Interestingly, this transfer of control would come at the cost of an efficiency loss in the exercise of control by the patient party if the parties can only engage in cheap talk. As we explain in section 4.2, $A$ might then always claim that the risky action is optimal no matter what he learns about the true underlying payoffs. There would then be imperfect communication between the parties and therefore learning about payoffs would be slower. Our assumption that the parties can communicate hard information thus plays an important role here.
} 
As before, Assumptions A6.b and A6.c ensure that there is no opportunity cost in thinking ahead and delaying investment for $B$. At the same time, under Assumption A6.a $A$ 's preferred course of action remains investing immediately. However, although $A$ makes contract proposals, the bargaining power is effectively with $B$, who can credibly reject all offers until after underlying payoffs are known. In this situation the preferred outcome for $A$ is to invest immediately and choose action $r$ without thinking, while the worst outcome is to invest immediately and think after the realization of $\theta_{2}$. As for $B$, she prefers to think ahead to any other alternative. Remarkably, $B$ in this situation fully gets her way even though she has no bargaining power.

Proposition 4: Under Assumptions A1, $\boldsymbol{A} 2$ and $\boldsymbol{A 6}$ agent $A$ has no choice but to follow B's preferred path of action, which is to invest only after payoffs in state $\theta_{2}$ has been uncovered. When this is the case the equilibrium contract is either $C_{R}$ when $R_{k}=\bar{R}_{k}$ or $C_{S}$ when $R_{k}=\underline{R}_{k}$.

\section{Proof: See the Appendix}

Interestingly, the equilibrium outcome of the contracting game may in some sense be inefficient, as the agents' joint welfare may be lower than under $A$ 's most preferred course of action. The reason is that $B$ does not internalize $A$ 's opportunity cost of delayed investment and $A$ is unable to compensate $B$ to get her to accept $A$ 's preferred action.

\section{Conflicting Underlying Objectives}

How do conflicts over action choice affect equilibrium contracting? In first generation models of incomplete contracting-in which contractual incompleteness is exogenously fixedallocating control to the right agent is more important the more agents disagree on the choice of action (Aghion and Bolton, 1992). In our setting instead, greater conflicts between agents are resolved through more complete contracts that generally give less discretion to the party in control. In other words, control is less relevant when there are greater conflicts between the parties. There are three related reasons why equilibrium contracts leave less room for discretion.

First, note that control rights may be as much a form of protection to the holder of the rights as a threat to the non-controlling party. Each party is concerned about potential 
abusive exercise of control by the other party and therefore may prefer to negotiate specific contractual guarantees rather than give up control.

Second, when the agents have conflicting goals they are likely to miscommunicate. That is, each party will have incentives to suppress information detrimental to itself but beneficial to the other party. As a result, the agents will learn more slowly as a group and the value of information is reduced. The lower value of information in turn induces the contracting parties to do less fine-tuning and to write coarser but more complete satisficing contracts.

Third, because the holder of control rights may have to accept limits on the exercise of control (to get the other party to participate) the value of control is reduced. Therefore, again, agents may prefer to sign a coarse and more complete contract rather than leaving the choice of actions in some states of nature open to be determined later.

Note finally that the agents are less likely to miscommunicate as long as they have not signed any contract and are free to walk away from the deal. The reason is simply that they are then less concerned about disclosing information that might be used against them. For these reasons, anticipated future conflicts introduce a bias towards thinking ahead or no thinking at all, which leads to more complete satisficing contracts.

To focus on the consequences of non-congruent underlying objectives we assume that the agents always disagree on which action is best under complete information:

Assumption A7: $\bar{R}_{A}<S_{A}<\underline{R}_{A}$ and $\bar{R}_{B}>S_{B}>\underline{R}_{B}$.

Although the agents disagree on what to do under complete information we shall for the most part continue to assume that their ex-ante expected payoffs remain aligned. That is, we shall maintain Assumption A1, which states that both agents prefer the risky action over the safe action under their common prior beliefs $\nu{ }^{11}$

\subsection{Miscommunication and the lower value of control}

Consider first the situation where the conflict is so severe that each party is better off liquidating the venture ex post than have its least preferred action chosen. Moreover, one party's least preferred action is the other party's most preferred action. Such a situation arises whenever the following assumption holds:

Assumption A8: $\bar{R}_{A}<0$ and $\underline{R}_{B}<0$.

\footnotetext{
${ }^{11}$ Note that assumptions A1 and A7 can only be consistent for interior values of prior beliefs $\nu$.
} 
We shall refer to this situation as one where the exercise of control is abusive. The reason is that Assumptions $\mathbf{A} \mathbf{7}$ and $\mathbf{A} \mathbf{8}$ together imply that any party in control in state $\theta_{2}$ takes an action that produces a negative payoff for the non-controlling party, a worse outcome than inaction which yields a zero payoff.

Recall that when both agents have congruent underlying objectives the net value for agent $k$ of determining the optimal action choice in state $\theta_{2}$ (when this choice is left to be determined after state $\theta_{2}$ arises) is given by

$$
\widehat{\Lambda} \rho_{k}^{*}-\rho_{k}
$$

This value can be obtained through the parallel thinking efforts of the two agents combined with full communication of any information obtained by the two agents.

When there are underlying conflicts among the two agents, the value of thinking before acting in state $\theta_{2}$ is generally lower, as the agents no longer "share all their thoughts" when they have conflicting goals and stop fully communicating what they have learned.

\subsubsection{The Dynamics of Communication and Learning}

The non-controlling party is understandably reluctant to disclose information to the controlling party that could be used against her. Thus, consider the situation the agents face under contract $C_{A}$ when they are uninformed in state $\theta_{2}$.

Suppose first that $B$ learns $R_{k}=\underline{R}_{k}$. She then prefers to postpone the time when the risky action is chosen by $A$ (as she then gets a discounted negative payoff $\underline{R}_{B}$ ). She therefore suppresses this information and reports that she did not learn anything. Hence, $A$ can only discover this payoff on his own with probability $\lambda_{A}$. Note, however, that if $A$ learns nothing after one round of thought experimentation (with probability $1-\lambda_{A}$ ) and $B$ reports nothing, $A$ updates his estimate of $\operatorname{Pr}\left(R_{k}=\underline{R}_{k}\right)$ from his prior $(1-\nu)$ to

$$
1-\nu_{1} \equiv \frac{(1-\nu)}{\nu\left(1-\lambda_{B}\right)+(1-\nu)} .
$$

Similarly, after $\tau$ rounds of experimentation in which $A$ has learned nothing and $B$ has reported nothing, $A$ 's posterior belief that $R_{k}=\underline{R}_{k}$ becomes

$$
1-\nu_{\tau} \equiv \frac{(1-\nu)}{\nu\left(1-\lambda_{B}\right)^{\tau}+(1-\nu)},
$$

which converges to 1 . Clearly then, after a sufficiently long run of unsuccessful trials it is optimal for $A$ to stop experimenting and to choose the risky action. 
Agent $A$ 's optimal stopping time $\tau_{A}$ and posterior $\nu_{\tau_{A}}$ are such that $A$ is indifferent between taking the risky action immediately or continuing thinking for one more round:

$$
\begin{gathered}
\nu_{\tau_{A}} \bar{R}_{A}+\left(1-\nu_{\tau_{A}}\right) \underline{R}_{A}=\delta\left[\nu_{\tau_{A}} S_{A} \Lambda+\left(1-\nu_{\tau_{A}}\right) \lambda_{A} \underline{R}_{A}+\right. \\
\left(\left(\nu_{\tau_{A}}(1-\Lambda)+\left(1-\nu_{\tau_{A}}\right)\left(1-\lambda_{A}\right)\right)\left(\nu_{\tau_{A}+1} \bar{R}_{A}+\left(1-\nu_{\tau_{A}+1}\right) \underline{R}_{A}\right)\right]
\end{gathered}
$$

(we ignore integer constraints for simplicity).

Although $B$ does not disclose anything $A$ still learns something from $B$ 's silence. Even if $A$ cannot tell for sure what the true payoff of the risky action is, when his beliefs have been updated to the point where he estimates that the risky action yields a payoff $\bar{R}_{A}$ with probability $\nu_{\tau_{A}}$, agent $A$ is better off stopping to think further and choosing the risky action.

Suppose next that $B$ learns $R_{k}=\bar{R}_{k}$. Although $B$ is less reluctant to disclose this information-for then $A$ responds by choosing the safe action with a positive payoff $S_{B}$ for $B$-she may still choose to withhold that $R_{k}=\bar{R}_{k}$. The reason is that by withholding this information she may be able to induce $A$ to choose the risky action (a preferred choice as $\bar{R}_{B}>S_{B}$ under Assumption A7). More precisely, when $A$ 's beliefs are near $\nu_{\tau_{A}}$ (so that $A$ prefers to stop if he remains uninformed about the optimal action) $B$ is better off withholding the information that $R_{k}=\bar{R}_{k}$. But when $A$ 's belief $\nu$ is small, then $A$ prefers to continue thinking when uninformed, in which case $B$ 's best response is to disclose $\bar{R}_{k}$, so as to bring forward the time when $A$ chooses the safe action.

Thus, the strategic difficulty in this situation is that $B$ 's best response varies with $A$ 's belief $\nu$. Moreover, $A$ 's best response also depends on $B$ 's disclosure policy. When $A$ 's belief is near $\nu_{\tau_{A}}, A$ prefers to stop thinking if he expects $B$ to disclose $R_{k}=\bar{R}_{k}$, but to continue thinking if he expects $B$ to withhold $R_{k}=\bar{R}_{k}$.

In short, a pure strategy equilibrium in stopping times and disclosure strategies cannot exist in this situation. However, as the next lemma establishes, a mixed strategy equilibrium always exists.

We denote by $\widehat{\tau}$ the first time at which $A$ 's beliefs $\nu_{\widehat{\tau}}$ are such that $A$ prefers to continue thinking if $B$ stops disclosing $\bar{R}_{k}$ at time $\widehat{\tau}-1$, but to stop if $B$ only stops disclosing $\bar{R}_{k}$ at time $\widehat{\tau}$.

Lemma 2: Under Assumptions A7 and A8, when the agents are uninformed in state $\theta_{2}$ the only equilibrium under contract contract $C_{A}$ that exists is a mixed strategy equilibrium, where both $A$ randomizes between stopping and continuing to think and $B$ randomized between disclosing and stopping to disclose $\bar{R}_{k}$ at any time $\tau \geq \widehat{\tau}+1$. 
Proof: See the Appendix.

\subsection{Coarse Contracts}

As the value of thinking before acting in state $\theta_{2}$ is lower when there are underlying conflicts among the two agents, the ex-ante value of leaving decisions to be fine-tuned when the state of nature arises is also lower. The implication, as we show here, is that the two agents may prefer not to fine-tune their action choice and to write a complete contract with a coarse action-plan. We refer to such contracts as coarse contracts.

Given that the agents do not cooperate in thinking through the best plan of action in state $\theta_{2}$ the value of control for $A$ under contract $C_{A}$ is now no more than

$$
\begin{aligned}
V^{A} \equiv & \sum_{\tau=1}^{\widehat{\tau}}\left[\nu S_{A} \Lambda\left(1+(1-\Lambda)^{\tau} \delta^{\tau}\right)+(1-\nu) \lambda_{A} \bar{R}_{A}\left(1+\left(1-\lambda_{A}\right)^{\tau} \delta^{\tau}\right)\right]+ \\
& \delta^{\widehat{\tau}}\left(\nu_{\widehat{\tau}} \bar{R}_{A}+\left(1-\nu_{\widehat{\tau}}\right) \underline{R}_{A}\right) .
\end{aligned}
$$

(note that since $A$ is indifferent between stopping or continuing thinking at $\widehat{\tau}$ his payoff at $\widehat{\tau}$ is given by $\left.\nu_{\widehat{\tau}} \bar{R}_{A}+\left(1-\nu_{\widehat{\tau}}\right) \underline{R}_{A}\right)$.

The main difficulty in our analysis here is the characterization of the first stopping time $\widehat{\tau}$. However, for some parameter values we can establish that $A$ prefers to stop thinking right away -so that $\widehat{\tau}=0$ - even if thinking is optimal if the agents cooperate. This is so if:

$$
\rho_{A} \geq\left(\nu S_{A} \Lambda+(1-\nu) \underline{R}_{A} \lambda_{A}\right)+\delta\left[\nu(1-\Lambda)+(1-\nu)\left(1-\lambda_{A}\right)\right] \rho_{A},
$$

which can rewritten as:

$$
\rho_{A} \geq \frac{\nu S_{A} \Lambda+(1-\nu) \underline{R}_{A} \lambda_{A}}{1-\delta\left(1-\left(\nu \Lambda+(1-\nu) \lambda_{A}\right)\right)} .
$$

To side-step the difficulty of characterizing the optimal first stopping time $\widehat{\tau}$ in general, we restrict attention to parameter values for which $\widehat{\tau}=0$. Thus, we assume henceforth,

Assumption A9: $\quad \rho_{A} \geq \frac{\nu S_{A} \Lambda+(1-\nu) \underline{R}_{A} \lambda_{A}}{1-\delta\left(1-\left(\nu \Lambda+(1-\nu) \lambda_{A}\right)\right)} \cdot{ }^{12}$

\footnotetext{
${ }^{12}$ Note that, since

$$
\left(\nu S_{A} \Lambda+(1-\nu) \underline{R}_{A} \lambda_{A}\right)+\delta\left[\nu(1-\Lambda)+(1-\nu)\left(1-\lambda_{A}\right)\right] \rho_{A}<
$$$$
\Lambda\left(\nu S_{A}+(1-\nu) \underline{R}_{A}\right)+\delta(1-\Lambda) \rho_{A},
$$

there are situations under assumption $\mathbf{A} 8$ where both $\widehat{\Lambda} \rho_{A}^{*}>\rho_{A}$ and condition (5) hold.
} 
Recall that when assumption A4 holds (that is $\widehat{\Lambda} \rho_{k}^{*}>\rho_{k}$ and $I<\frac{\delta \pi}{2}$ ) and when the agents have congruent underlying preferences, then satisficing contracts are incomplete and assign control to one or both agents (Proposition 2). In contrast, as we show below, when the agents have non-congruent preferences they will sign a complete but coarse contract under the same circumstances.

We have already established that under Assumption A9, A cannot do better under contract $C_{A}$ than under contract $C_{R}$. Similarly, when the agents sign contract $C_{A B}$ in this situation, the best they can do is not to think and instead immediately choose the risky action in state $\theta_{2}$. The value of thinking is even lower than under contract $C_{A}$, as a complete stalemate arises once the agents know the true payoff $R_{k}$ and learn that they fundamentally disagree on the action choice. Therefore an additional reason why the risky action is chosen when the agents do not know the true payoff $R_{k}$ is that each party knows that by engaging in thought experimentation they run the risk of a stalemate.

Proposition 5: Under Assumptions A1, A4, $\boldsymbol{A 7}, \boldsymbol{A 8}$ and $\boldsymbol{A 9}$ agent $A$ (weakly) prefers the coarse contract $C_{R}$ at date 0 to contracts $C_{A}, C_{B}$ or $C_{A B}$, and therefore there is no equilibrium in which an incomplete contract is proposed and accepted.

Proof: See the Appendix.

Under the assumptions of Proposition 5, incomplete contracts cannot be equilibrium contracts. This is entirely due to the agents' extremely non-congruent objectives. As we have shown in the previous section, if the agents had congruent preferences then under Assumption A4 they would prefer to sign an incomplete contract. Here, the agents prefer to commit to a coarse action-plan rather than leave things to be fine-tuned later, as they anticipate that they will face ex-post miscommunication inefficiencies. Miscommunication, in turn, reduces the value of thinking and fine-tuning ex post to the point that the agents prefer not to think and instead to stick to a coarse action plan.

Note that, although incomplete contracts are (weakly) dominated here, it is not obvious whether a complete coarse contract or a complete state-contingent contract is optimal. More precisely, it is not clear whether the agents will immediately sign contract $C_{R}$ or whether they will think first and then sign a complete state-contingent contract. Indeed, despite their non-congruent objectives the agents may prefer to put their differences aside and collaborate in working out a state-contingent action-plan before committing to invest. 
Agent $B$ prefers the coarse contract $C_{R}$, since under a state-contingent action-plan $A$ imposes the risky action when it is detrimental to $B$ and the safe action when $B$ stands to benefit from the risky action:

$$
-I+\frac{\delta \pi}{2}+\frac{\delta \rho_{B}}{2}>\widehat{\Lambda}\left[\nu\left(-I+\frac{\delta \pi}{2}+\frac{\delta S_{B}}{2}\right)+(1-\nu) \max \left\{0,-I+\frac{\delta \pi}{2}+\frac{\delta \underline{R}_{B}}{2}\right\}\right] .
$$

Agent $A$ also prefers the coarse contract $C_{R}$ if the opportunity cost of delaying investment $\left(\frac{\delta \pi}{2}-I\right)$ is high, and otherwise prefers to think before investing.

Suppose that B's participation constraint does not bind:

Assumption A10: $-I+\frac{\delta \pi}{2}+\frac{\delta \underline{R}_{B}}{2}>0$.

As we have established in the previous section, under Assumptions A4 and A10, agents with congruent preferences agree on the incomplete contract $C_{A}$. In contrast, here, with extreme non-congruent preferences such that Assumption $\mathbf{A 8}$ holds, either the coarse contract $C_{R}$ is signed at date 0 , or both agents think ahead before signing a contract.

Proposition 6: Under Assumptions A1, A4, A7, A8, A9 and A10, a cutoff $\bar{I}<\frac{\delta \pi}{2}$ exists such that in equilibrium agents think ahead before committing to invest in the venture if $I \geq \bar{I}$. Otherwise, $A$ and $B$ sign the coarse contract $C_{R}$ at date 0 .

Proof: See the Appendix.

The agents prefer to agree on a complete contract, because their conflicting objectives undermine future cooperation and, thus, reduce the benefits of postponing some decisions. At the same time, the allocation of control rights is too blunt an instrument, since the controlling party can exploit the non-controlling party.

Note finally that when Assumption A9 does not hold a complete contract can still emerge (provided $I$ is large enough) even though the agents would have chosen an incomplete contract had their objectives been congruent.

\subsection{The need to compromise reduces the value of planning ahead}

In this section, we consider a situation where the agents are best off thinking ahead of investing. We show that there will be no miscommunication in equilibrium as long as the agents are not committed to the venture. Nevertheless, the need to compromise to make 
the venture acceptable to both agents may reduce the value of information sufficiently that the agents end up preferring a coarse, complete contract to a complete state-contingent contract. Similarly, the need to reduce discretion of the controlling party may reduce the value of control sufficiently that the agents prefer a coarse complete contract.

Under Assumption A3 ( $\widehat{\Lambda} \rho_{k}^{*}>\rho_{k}$ and $\left.I>\frac{\delta \pi}{2}\right)$ each agent would prefer to think before choosing an action, provided it could choose its preferred action and agents share their thoughts. In addition, since $I>\frac{\delta \pi}{2}$, each agent would prefer this thinking to take place ahead of investing. Under conflicting preferences, however, agents cannot both implement their preferred action. Only the controlling party may be able to do so. Even though Assumption A3 holds, the non-controlling party may well prefer not to engage in any planning. To get the non-controlling party to accept a contract where it has no control, the controlling party may then need to constrain its own discretion. But as we show below, while limiting its discretion the controlling party may in turn prefer not to engage in any planning and instead sign a complete but coarse contract.

To illustrate this observation we assume that the conflict among the agents is so severe that, if the agents think ahead of investing and learn that $R_{k}=\underline{R}_{k}, B$ prefers to stay out of the venture rather than agreeing to $A$ 's preferred action:

Assumption A11: $-I+\frac{\delta \pi}{2}+\frac{\delta}{2} \underline{R}_{B}<0$.

Here, to get $B$ to participate $A$ must commit not to implement the risky action in state $\theta_{2}$ with a probability exceeding $x^{*}$ given by:

$$
-I+\frac{\delta \pi}{2}+\frac{\delta}{2}\left(x^{*} \underline{R}_{B}+\left(1-x^{*}\right) S_{B}\right)=0 .
$$

Note that, unlike in the situation when the parties have already made their investments and are uninformed in state $\theta_{2}$, there will be no miscommunication when the agents think before they commit to the venture. The reason is that the worst outcome for either party before investment takes place is to obtain a payoff of zero by walking away from the venture. Therefore, a (weak) best response for $B$ is to always disclose $\underline{R}_{k}$. And given that $B$ always discloses $\underline{R}_{k}$, it is also a best response to always disclose $\bar{R}_{k}$. The reason is that if $B$ does not disclose $\bar{R}_{k}, A$ only stops learning when his beliefs $\nu_{\tau}$ are sufficiently close to 1 , in which case he chooses the safe action. But then $B$ is only delaying the time when she obtains $S_{B}$ by not disclosing $\bar{R}_{k}$.

Still, even though agents share their thoughts, the value of information may be sufficiently reduced for $A$ (when he needs to compromise), that he prefers to immediately settle on the 
risky action without thinking. If $A$ ends up choosing the safe action most of the timewhenever $R_{k}=\bar{R}_{k}$, and with probability $\left(1-x^{*}\right)$ when $R_{k}=\underline{R}_{k}$-what is the point of engaging in time-consuming thinking?

Another difficulty here is that after one period of thinking the agents bargain under incomplete information. Thus, if $A$ makes an offer $C_{R}$ in any period $t \geq 1, B$ may suspect that $A$ actually knows that $R_{k}=\underline{R}_{k}$.

Proposition 7: Under Assumptions A1, A3, A7, A8, A9 and A11, an offer $C_{R}$ from $A$ at $t=0$ is an equilibrium of the contracting game when

$$
x^{*}=\frac{1}{\underline{R}_{B}-S_{B}}\left(\frac{2 I}{\delta}-\pi-S_{B}\right)
$$

is close enough to zero.

Proof: See the Appendix.

When agents attempt to write down a detailed plan of action, they also learn that they have fundamental differences. The need to compromise then reduces the value of information and will result in less fine-tuned contracts. A coarse contract is also a compromise but one where the cost of thinking is avoided. Here a deal is quickly concluded because this is an efficient resolution of the agents conflicting objectives, avoiding lengthy and ultimately sterile negotiations.

\subsection{Preliminary contracts}

So far we have restricted attention to five main contracts. But there is also a sixth contract, which we refer to as a preliminary contract which can be an equilibrium contract. Under this contract, which we denote by $C_{\alpha}$, the agents agree to first think ahead of investing and are committed to an action contingent on $R_{k}$.

This contract may be preferred to the coarse contract $C_{R}$ because it yields higher expected payoffs by committing the agents to participate even when an agent's ex-post participation constraint does not hold. More precisely, the preliminary contract can secure $B$ 's participation ex ante, and thus relax the ex-post participation constraint,

$$
-I+\frac{\delta \pi}{2}+\frac{\delta\left(x S_{B}+(1-x) \underline{R}_{B}\right)}{2} \geq 0 .
$$

A preliminary contract can then raise $A$ 's value from thinking ahead while guaranteeing $B$ 's participation. However, to be acceptable to $B$ the preliminary contract must guarantee 
$B$ a sufficiently high expected payoff in state $\theta_{2}$ even though $A$ gets to choose the risky action with a higher probability than $x^{*}$.

To illustrate this possibility while keeping the analysis simple we shall consider the special situation where $B$ is unable to think, so that $\lambda_{B}=0 .{ }^{13}$

Consider the following preliminary contract $C_{\alpha}$ offered by $A$ to $B$ at $t=0$ :

a) the agents commit to invest once they have discovered the value of $R_{k}$;

b) if $R_{k}=\underline{R}_{k}$, action $r$ is chosen in state $\theta_{2}$;

c) if $R_{k}=\bar{R}_{k}$, action $s$ is chosen with probability $\chi$ and action $r$ with probability $(1-\chi)$ in state $\theta_{2}$, where $\chi$ solves agent $B$ 's participation constraint at date 0 .

We shall show that this contract may be strictly preferred by $A$ to $C_{R}$ and that $B$ accepts this offer under the assumptions of Proposition 7, but when

$$
x^{*}=\frac{1}{\underline{R}_{B}-S_{B}}\left(\frac{2 I}{\delta}-\pi-S_{B}\right)
$$

is close to 1 . Indeed, when $x^{*}$ is close to 1 the agents prefer to think ahead and settle on either contract $C_{S}$ or $C_{x^{*}}$ rather than immediately agree on $C_{R}$. But we shall show that in this case the agents can do even better by signing a preliminary contract under two additional assumptions.

This preliminary contract, offered before the agents have thought through their action in state $\theta_{2}$, allows them to effectively transfer payoffs across states of nature and thus achieve a higher ex-ante expected payoff, as with an insurance contract. Although they are both risk neutral, there are gains from such an agreement by letting the agents trade commitments to choosing the risky action in situations when it is not their most preferred action. In this way the agents can make ex-post non-transferable utilities partially transferable ex ante.

The role of a preliminary contract is, thus, to overcome a form of Hirshleifer effect, where information eliminates insurance or trading opportunities and thus results in a decline in exante utility. Here, as the agents' information changes over time, so does the intensity of the conflicts that oppose them. Absent a preliminary contract, $B$ will be unwilling to invest when it expects to get $\underline{R}_{B}$ in state $\theta_{2}$. Under the veil-of-ignorance concerning agents' true payoffs, they are able to find room for agreements they would not be able to reach once the information is revealed.

\footnotetext{
${ }^{13}$ When $\lambda_{B}>0$ agent $B$ 's thinking also contributes to the contracting parties aggregate learning capacity. In this situation the analysis is more complex as $B$ has incentives not to share her thoughts. The preliminary contract must then generally also specify a stopping time when the parties are committed to invest.
} 
Suppose that in addition to Assumptions A1, A3, A7, A8, and A11, the following additional assumptions hold:

Assumption A12: $\quad \frac{\underline{R}_{A}-S_{A}}{\bar{S}_{A}-\bar{R}_{A}}>\frac{S_{B}-\underline{R}_{B}}{\bar{R}_{B}-S_{B}}$,

and,

Assumption A13: $-I+\frac{\delta \pi}{2}+\frac{\delta}{2} \bar{R}_{A}>0$.

Then we are then able to establish:

Proposition 8: Under the same Assumptions as in Proposition 7 and Assumptions A12 and $\boldsymbol{A} \mathbf{1 3}$, and provided that

$$
x^{*}=\frac{1}{\underline{R}_{B}-S_{B}}\left(\frac{2 I}{\delta}-\pi-S_{B}\right)
$$

is sufficiently close to 1, the unique subgame-perfect equilibrium is such that:

i) A offers a preliminary contract to $B$ at $t=0$ with the following terms: a) the agents commit to invest once they have thought through $R_{k}$; b) if $R_{k}=\underline{R}_{k}$ then action $r$ is chosen in state $\theta_{2}$; c) if $R_{k}=\bar{R}_{k}$ then action $s$ is chosen with probability $\chi$ and action $r$ with probability $(1-\chi)$ in state $\theta_{2}$, where $\chi$ is given by:

$$
\left.-I+\frac{\delta \pi}{2}+\frac{\delta}{2}\left[\nu\left(\chi S_{B}+(1-\chi) \bar{R}_{B}\right)+(1-\nu) \underline{R}_{B}\right)\right]=0
$$

ii) both agents think ahead and share their thoughts.

Proof: See the Appendix.

Under Assumption A12, $A$ strictly prefers the preliminary contract to thinking ahead and settling on either contracts $C_{S}$ or $C_{x^{*}}$. Moreover, under assumption $\mathbf{A} \mathbf{3}$ both agents prefer the preliminary contract to $C_{R}$ given that $x^{*}$ is close to 1 . Finally, under the contract both agents prefer to think and share their thoughts, as no investment can take place unless they discover value of $R_{k}$.

\section{Extensions}

This section explores two extensions to our basic setup. 


\subsection{Cheap talk}

We have assumed that agents' thoughts are hard information. This may be realistic in some situations (e.g. a mathematical proof) but less so in others. Here we examine the opposite case in which thoughts are soft information so that communication is pure cheap talk. Most of our qualitative results extend to this case.

With cheap talk there is inevitably more miscommunication than with disclosure of hard information. First, when agents disagree about how cautiously to proceed there is now some miscommunication. Second, under extreme non-congruent preferences over actions miscommunication is now total, while before it was only partial.

Under congruent objectives, however, agents trust each others's reports and communication is unimpaired. A result similar to Lemma 1, with the addition of Assumption A3, can be established, and Propositions 1 and 2 continue to hold.

Thus, consider first the situation where the agents may disagree on how cautiously to proceed. We show below that even though cheap talk allows for miscommunication, a result analogous to Proposition 3 and Corollary 1 obtains under slightly different conditions.

The problem under cheap talk is that the more patient agent can no longer trust the more impatient one to tell the truth. Since the impatient agent prefers to choose the risky action without thinking further, it will always pretend that it has found that the risky action has a payoff $\bar{R}_{k}$ when it has not discovered anything. The impatient agent is thus credible only when reporting $R_{k}=\underline{R}_{k}$.

Suppose again that $A$ is impatient and prefers the risky action in state $\theta_{2}$ without thinking further, while $B$ prefers to think before acting. Miscommunication then has two opposing effects. On the one hand, $B$ 's threat to reject all offers until she has thought through $R_{k}$ is less credible, because miscommunication slows down the agents' joint thinking, thus reducing the value of thinking ahead. On the other hand, the value of control for $B$ is reduced for the same reason. On net, although $B$ is more likely to accept a contract without control rights, when she does require control to sign on, she demands more control than when thoughts are hard information.

More formally, assume that beginning at date $t=0, A$ makes repeated offers of $C_{R}$, which $B$ rejects to gain time to think about $R_{k}$. Following each rejection both agents think and engage in cheap talk. As we have observed, $A$ reports $\bar{R}_{k}$ both when this is the true payoff and when he learns nothing, and he truthfully reports $\underline{R}_{k}$. As for $B$, she truthfully shares her thoughts. 
Therefore, when $A$ reports $\underline{R}_{k}, B$ believes this is true and accepts $A$ 's offer $C_{S}$. In contrast, when $A$ reports $\bar{R}_{k}, B$ only updates her belief $\nu_{t}$. After $t$ rounds of communication of $\bar{R}_{k}$, her posterior belief is:

$$
\nu_{t}=\frac{\nu}{\nu+(1-\nu)\left(1-\lambda_{A}\right)^{t}} .
$$

Thus, $B$ 's beliefs $\nu_{t}$ converge to 1 . In other words, it dawns on $B$ that $A$ has learned that $R_{k}=\bar{R}_{k}$. Following a sufficiently long sequence of announcements of $\bar{R}_{k}, B$ therefore finds it optimal to stop rejecting $A$ 's offers of $C_{R}$. At her optimal stopping time, denoted by $t_{B}, B$ is indifferent between accepting $C_{R}$ and and thinking for one more period. That is, her posterior $\nu_{t_{B}}$ is such that:

$$
\begin{gathered}
\nu_{t_{B}} \bar{R}_{B}+\left(1-\nu_{t_{B}}\right) \underline{R}_{B}=\delta\left[\nu_{t_{B}} \lambda_{B} \bar{R}_{B}+\left(1-\nu_{t_{B}}\right) S_{B} \Lambda+\right. \\
\left(\left(\nu_{t_{B}}\left(1-\lambda_{B}\right)+\left(1-\nu_{t_{B}}\right)(1-\Lambda)\right)\left(\nu_{t_{B}+1} \bar{R}_{B}+\left(1-\nu_{t_{B}+1}\right) \underline{R}_{B}\right)\right] .
\end{gathered}
$$

Although conflicts over cautiousness may be reduced if miscommunication slows down thinking, they do not disappear. Formally, Assumption A5 must be replaced by Assumption A5b below to reflect the change in expected discounted payoffs resulting from miscommunication. Denoting agent $k$ 's payoff $(k=A, B)$ when both agents think before choosing the optimal action in state $\theta_{2}$ by:

$$
\begin{aligned}
W^{k} \equiv & \sum_{t=1}^{t_{B}}\left[\nu \bar{R}_{k} \lambda_{B}\left(1+\left(1-\lambda_{B}\right)^{t} \delta^{t}\right)+(1-\nu) S_{k} \Lambda\left(1+(1-\Lambda)^{t} \delta^{t}\right)\right]+ \\
& \delta^{t_{B}}\left(\nu_{t_{B}} \bar{R}_{k}+\left(1-\nu_{t_{B}}\right) \underline{R}_{k}\right),
\end{aligned}
$$

then Assumption A5b is as follows:

Assumption A5b: $W^{A}<\rho_{A}, W^{B} \geq \rho_{B}$ and $I<\frac{\delta \pi}{2}$

Denoting by $Y^{B}$ agent $B$ 's payoff when both agents think ahead of investing:

$$
\begin{aligned}
Y^{B} \equiv & \sum_{t=1}^{t_{B}}\left\{\nu\left[-I+\frac{\delta \pi}{2}+\frac{\delta \bar{R}_{B}}{2}\right] \lambda_{B}\left(1+\left(1-\lambda_{B}\right)^{t} \delta^{t}\right)+\right. \\
& \left.(1-\nu)\left[-I+\frac{\delta \pi}{2}+\frac{\delta S_{B}}{2}\right] \Lambda\left(1+(1-\Lambda)^{t} \delta^{t}\right)\right\}+ \\
& \delta^{t_{B}}\left[-I+\frac{\delta \pi}{2}+\frac{\delta\left(\nu_{t_{B}} \bar{R}_{B}+\left(1-\nu_{t_{B}}\right) \underline{R}_{B}\right)}{2}\right],
\end{aligned}
$$


then we are able to establish the following analog of Proposition 3 for the contracting game with cheap talk.

Proposition 3b Under Assumptions A1, A2, A5b and under condition

$$
-I+\frac{\delta \pi}{2}+\frac{\delta \rho_{B}}{2}<Y^{B}
$$

in equilibrium $A$ offers immediately $C_{B}$ with probability $y^{*}$ and $C_{R}$ with probability $\left(1-y^{*}\right)$, where $y^{*}$ is given by:

$$
-I+\frac{\delta \pi}{2}+y^{*} W^{B}+\left(1-y^{*}\right) \frac{\delta \rho_{B}}{2}=Y^{B} .
$$

When the reverse condition holds, $A$ immediately offers contract $C_{R}$.

\section{Proof: Omitted.}

Similarly, a result analogous to Proposition 4, which we omit, can be established under modified conditions to reflect miscommunication.

Now consider situations where the two agents disagree about the choice of action (Assumption A7 holds). In this case, communication breaks down completely, as each agent has a strict incentive to mislead the other. As a result, the two agents think by themselves and duplicate their cognitive efforts. Results analogous to Propositions 5, 6 and 7 obtain again under modified conditions to account for the slower thinking. The key difference with the previous analysis is that the parameter region for which equilibrium contracts are coarse is larger.

Lemma 3: When the equilibrium contract under hard information is a coarse contract, it is also the equilibrium contract when information is soft.

Proof: The agents' payoffs under a coarse contract are the same whether information is hard or soft. Indeed, no thinking is involved. Moreover, any other contract offer that involves some thinking cannot result in a higher payoff for $A$ under soft information than under hard information. It therefore follows that if $C_{R}$ is an equilibrium offer at $t=0$ when information is hard it must also be an equilibrium offer when information is soft.

Finally, another difference with the case with hard information is that negotiations may last forever even when $A$ has thought through $R_{k}$. Indeed, by repeating over and over again the same information, $A$ is unable to persuade $B$ that his information is reliable. Since $B$ 's beliefs do not change, $A$ does not have a finite stopping time. This, in turn, reduces the value of a preliminary contract. 


\subsection{Transferable utility}

We assume now that utility is fully transferable through (state-contingent) monetary payments. In this case, a preliminary contract has even greater benefits. Indeed, by first specifying the broad terms of the deal, such a contract aligns the agents' objectives, leading them to agree on how much thinking should precede investment. Hence, they will more readily accept to invest without having worked out a complete action-plan.

Lemma 4 (The Congruence Principle): It is weakly optimal for the agents to sign a preliminary contract establishing how the agents will share the profits.

\section{Proof: See the Appendix.}

Once the agents have agreed on profit-sharing, their objectives are fully congruent, and communication is perfect whether thoughts are hard or soft information. Therefore, the contracting problem reduces to determining the team's optimal plan of action. Any actions that the contracting agents determine by thinking ahead will be specified in the contract and any decisions to be determined at the time when they arise will be taken jointly by the two agents.

Strictly speaking, there is no need for contracts beyond the preliminary agreement. Note, however, that under even a very small risk of change in one of the agents' preferences resulting in a conflict ex-post, the agents would strictly prefer to explicitly spell out what future action choices they have agreed to in a contract. For the same reason the agents would strictly prefer to specify a governance structure that defines the process by which future decisions are taken.

With only one state of nature $\left(\theta_{2}\right)$ where some thinking is needed, the contracting problem with transferable utility collapses to a simple decision problem of the type studied in Bolton and Faure-Grimaud (2008). When thinking is required in more than one state of nature (e.g. in state $\theta_{1}$ ) the team problem is of independent interest as it raises a number of organizational design questions. For instance, an interesting set of questions is who in the team should think about what problem? Is the team more efficient when both agents think about the same problem at the same time, or rather when they specialize and engage in parallel thinking? The analysis of these issues is beyond the scope of this paper and is left for future research. 


\section{Conclusion}

We have proposed and explored a first contracting model between two agents facing timedeliberation costs. In this model, equilibrium contracts may be endogenously incomplete. Control rights assigned to one of the parties allow the controlling agent to defer timeconsuming deliberations to a later date without exposing her to too much uncertainty. As she will be in charge of the decisions most critical to her, she need to worry too much and unduly prolong negotiations at the initial contracting stage.

However, when agents face potentially major conflicts they tend to resolve these up-front, by writing more complete initial contracts. This more complete contract may be either a more state-contingent or a coarser contract. Thus conflicts among contracting agents tend to result in excessively complete contracts from the perspective of joint payoff maximization.

Equilibrium contracts in our model are incomplete for two reasons: first, the costs of thinking about how to complete them may exceed the expected benefits; and second, the costs of thinking about how to outwit the other agent also exceed the expected benefits. In contrast to first generation incomplete contracting models, contracts are not incomplete due to exogenously given enforcement constraints. Indeed, we have assumed that all statecontingent transactions are fully enforceable. Instead, contractual incompleteness is due to the limited imagination of the contracting agents.

We believe that this set-up lends itself naturally to the analysis of contractual innovation. Over time new contracting agents can learn from past contracts and may innovate relative to existing contracts, even if the legal environment remains unchanged. Also, in our setup the law has other roles than just an enforcement role, and contract law does not simply reduce to the enforcement of a freedom of contracting regime. Courts and trade associations can add value by specifying default rules or contracts, which effectively reduce transaction costs for new contracting agents. Indeed, Chakraborty and MacLeod (2008) argue that the American Institute of Architects standard form contracts for construction projects, which have evolved over a long period of time, indeed provide an efficient default contract for complex construction projects that is readily available to any contracting parties in the construction business. They also show that a central feature of these contracts is the inclusion of control rights and governance structures that determine the ex post bargaining power of parties.

Similarly, by putting constraints on the exercise of control rights, courts can reduce the risk of abusive control and thereby allow contracting agents to sign more efficient incomplete 
contracts. In the absence of such constraints the equilibrium outcome of the contracting game may be that both parties end up wasting a lot of time fine-tuning the contract before investing, while with legal limits on the exercise of control the parties may end up agreeing on an incomplete contract much more quickly. A fuller exploration of these implications and ideas are left for future research.

\section{References}

Aghion, P. and P. Bolton (1992) "An Incomplete Contracts Approach to Financial Contracting." Review of Economic Studies, 59, 473-94.

Al Najjar, N., L. Anderlini, and L. Felli (2002): "Unforeseen Contingencies," STICERD Discussion Paper TE/02/431, London School of Economics.

Anderlini, L. and L. Felli (1994) "Incomplete Written Contracts: Undescribable States of Nature", Quarterly Journal of Economics, 109 (4), pp. 1085-124.

Anderlini, L. and L. Felli (1999). "Incomplete Contracts and Complexity Costs", Theory and Decision, 46 (1), pp. 23-50.

Anderlini, L. and L. Felli, (2001): "Costly Bargaining and Renegotiation," Econometrica, $69,377-411$.

Bajari, P. and S. Tadelis, (2001) "Incentives versus Transaction Costs: A Theory of Procurement Contracts", RAND Journal of Economics, Vol. 32, No. 3, 387-407

Battigalli, P. and G. Maggi (2002) "Rigidity, Discretion and the Costs of Writing Contracts", American Economic Review, 92 (4), pp. 798-817.

Berry, D. A. and D. Fristedt (1985), Bandit Problems: Sequential Allocation of Experiments, London, Chapman Hall.

Bienz, C. and U. Walz (2008), "Venture Capital Exit Rights", mimeo Goethe University Frankfurt.

Bolton, P. and M. Dewatripont (2005), Contract Theory, Cambridge MA: MIT Press.

Bolton, P. and A. Faure-Grimaud (2008), "Thinking Ahead: The Decision Problem", forthcoming Review of Economic Studies.

Chakravarty S. and B. MacLeod (2008), "Contracting in the Shadow of the Law", Rand Journal of Economics, forthcoming

Conlisk, J. "Costly Optimizers Versus Cheap Imitators" Journal of Economic Behavior and Organization, 1980, 275-293. 
Conlisk, J. "Optimization Cost" Journal of Economic Behavior and Organization, 1988, $213-228$.

Conlisk, J. "Why bounded rationality?" Journal of Economic Literature, June 1996, 34 (2), pp.669-700.

Dye, R. (1985) "Costly Contract Contingencies", International Economic Review, 26 (1), pp. $233-50$.

Gittins, J.C. and D. M. Jones (1974), "A Dynamic Allocation Index for the Sequential Design of Experiments.", in Progress in Statistics, Gani, Sarkadi and Vince (eds.), New York, North Holland

Gittins, J.C. (1979) "Bandit processes and dynamic allocation indices", Journal of the Royal Statistical Society B, 41, 2, p.148-77.

Grossman, S.J. and O.D. Hart (1986). "The Costs and Benefits of Ownership: A Theory of Vertical and Lateral Integration." Journal of Political Economy, 94, 691-719.

Hart, O. D. (1995). Firms, Contracts, and Financial Structure. Clarendon Lectures in Economics. Oxford University Press.

Hart, O., and J. Moore. (1988) "Incomplete Contracts and Renegotiation." Econometrica, 56, 755-85.

Hart, O., and J. Moore. (2007), "Contracts as Reference Points", mimeo Harvard University

MacLeod, W. B. (2000): "Complexity and Contract," Revue d'Economie Industrielle, 92, $149-178$.

Kaplan, S., F. Martel, and P. Stromberg (2007), "How Do Legal Differences and Experience Affect Financial Contracts?," Journal of Financial Intermediation, forthcoming.

Maskin, E. and J. Tirole (1999): "Unforeseen Contingencies and Incomplete Contracts," Review of Economic Studies, 66, 83-114.

Radner, R. (1975) "Satisficing", Journal of Mathematical Economics, 2 (2), 253-62.

Radner, R. and M. Rothschild (1975), "On the Allocation of Effort", Journal of Economic Theory, 10 (3), 358-76.

Rothschild, M. (1974) "A Two-Armed Bandit Theory of Market Pricing," Journal of Economic Theory, 9, 185-202.

Simon, H. "A behavioral model of rational choice," Quarterly Journal of Economics, 1955, 69 (1).

Tirole, J. (1999) “Incomplete Contracts: Where Do We Stand?" Econometrica, 67 (4), 
pp. $741-81$.

Tirole, J. (2008) "Bounded Rationality and Incomplete Contracts", forthcoming American Economic Review

Whittle, P. (1980) "Multi-armed Bandits and the Gittins Index", Journal of the Royal Statistical Society, 42, 143-149

Whittle, P. (1982), Optimization over time: Dynamic Programming and Stochastic Control, John Wiley, New York

Williamson, O. (1979) "Transaction-Cost Economics:The Governance of Contractual Relations." Journal of Law and Economics, 22, 233-61.

Williamson, O. (1985). The Economic Institutions of Capitalism, New York: Free Press.

\section{APPENDIX}

Proof of Proposition 3: We first establish a series of preliminary results that simplify the argument.

Claim 1: Let $U_{\min }^{R F I}$ be the lowest payoff that the receiver can guarantee herself in any subgame under complete information. Then, either the proposer implements her most preferred contract or the receiver gets exactly $U_{\min }^{R F I}$.

Proof: Observe first that $U_{\min }^{R F I}=0$ in the absence of a pre-existing contractual agreement. Suppose now that the claim is not true and that there exists an SPNE where under full information, the receiver gets some payoff $\widehat{U}>U_{\min }^{R F I}$, and the proposer is not offering his most preferred plan of action. For this to be true, it must be that the receiver rejects any offer that gives her less than $\widehat{U}$. But, given that the proposer is not making his most preferred offer, it must then be the case that the receiver is just indifferent between accepting and rejecting the offer giving her $\widehat{U}$. Otherwise, the receiver could offer a lottery that would put some weight $\varepsilon$ on his most preferred contract and $(1-\varepsilon)$ on the offer currently providing $\widehat{U}$ to the receiver. Therefore it must be that along the equilibrium path in such an equilibrium, at any date $t, \widehat{U}_{t}=\delta \widehat{U}_{t+1}$. Iteration of this argument requires $\widehat{U}_{t+\tau}$ to go to infinity as $\tau$ goes to infinity, which is impossible.

Claim 2: Denote by $U^{R F I}$ the unique subgame perfect equilibrium payoff that the receiver obtains in any subgame under complete information. Then, in any subgame where the payoffs 
of the risky action are unknown, either the proposer offers her most preferred contract, or the receiver gets $U_{\min }^{R}=\widehat{\Lambda} U^{R F I}$.

Proof: Suppose again this is not true. As in the proof of Claim 1, it then follows that the receiver must be indifferent at any date $t$ between accepting or rejecting the offer that gives the receiver some utility level $\widetilde{U}_{t}^{R}>U_{\min }^{R}$. In particular, it then must be the case that

$$
\widetilde{U}_{t}^{R}=\Lambda U^{R F I}+(1-\Lambda) \delta \widetilde{U}_{t+1}^{R}
$$

And, if $\widetilde{U}_{t}^{R}=\widetilde{U}_{t+1}^{R}$, then $\widetilde{U}_{t}^{R}=\frac{\Lambda}{1-(1-\Lambda) \delta} U^{R F I}=\widehat{\Lambda} U^{R F I}$, a contradiction. Alternatively, iterating the same the argument, we would find that

$$
\widetilde{U}_{t+\tau}^{R}=\frac{\widetilde{U}_{t}^{R}}{(1-\Lambda)^{\tau} \delta^{\tau}}-\widehat{\Lambda} U^{R F I} \frac{1-(1-\Lambda)^{\tau} \delta^{\tau}}{(1-\Lambda)^{\tau} \delta^{\tau}}
$$

which, when $\widetilde{U}_{t}^{R}>U_{\text {min }}^{R}$ requires $\widetilde{U}_{t+\tau}^{R}$ to go to infinity when $\tau$ goes to infinity. Again, this leads to a contradiction.

We now make use of these observations to establish Proposition 3.

Note first that under assumptions A2 and A4 party $B$ 's minimum guaranteed payoff is

$$
U_{\min }^{B}=\widehat{\Lambda}\left[-I+\frac{\delta \pi}{2}+\frac{\delta \rho_{B}^{*}}{2}\right]
$$

If condition (4) in Proposition 3 does not hold, so that

$$
I+\frac{\delta \pi}{2}+\frac{\delta \rho_{B}}{2} \geq \widehat{\Lambda}\left(-I+\frac{\delta}{2}\left(\pi+\rho_{B}^{*}\right)\right)
$$

then the proposer's most preferred contract- $C_{R}$-gives a higher expected payoff to $B$ than $U_{\min }^{B}$. Therefore $B$ 's best response is to accept this offer.

Now suppose that condition (4) holds. Then, from claim 1, the receiver gets exactly $U_{\text {min }}^{B}$ in equilibrium.

To complete the proof of proposition 3 it remains to show that the stochastic contract offer that gives $A$ the highest possible payoff while guaranteeing $U_{\min }^{B}$ to $B$, takes the form described in the proposition, namely that both agents agree to invest immediately, party $B$ gets control with probability $y^{*}$ and the risky action is chosen in state $\theta_{2}$ with probability $\left(1-y^{*}\right)$.

There are several types of stochastic contracts that can implement $U_{\min }^{B}$. A first contract is to give full control to party $B$ (draw contract $C_{B}$ ) with probability $y$ and to take the 
risky action in state $\theta_{2}$ with probability $(1-y) \cdot{ }^{14}$ An alternative offer is to give $B$ control in every period with some probability $z$ and to take the risky action in state $\theta_{2}$ with probability $(1-z)$. As we show below these two contracts are in fact equivalent. To see this, note that under the latter contract party $k$ expects to receive:

$$
\begin{gathered}
(1-z) \rho_{k}+z \Lambda \rho_{k}^{*}+ \\
z(1-\Lambda) \delta\left[z(1-\Lambda) \delta\left[(1-z) \rho_{k}+z \Lambda \rho_{k}^{*}+z(1-\Lambda) \delta[\ldots]\right]=\right. \\
\frac{z \Lambda}{1-z(1-\Lambda) \delta} \rho_{k}^{*}+\frac{1-z}{1-z(1-\Lambda) \delta} \rho_{k} .
\end{gathered}
$$

Now setting

$$
y=\frac{z \Lambda}{1-z(1-\Lambda) \delta}
$$

this reduces to

$$
y \widehat{\Lambda} \rho_{k}^{*}+(1-y) \rho_{k}
$$

(Note also that there is no loss of generality in considering only stationary strategies $z_{t}=z$ for all $t)$.

We now characterise the highest payoff available to $A$ under the constraint that $B$ gets $U_{\text {min }}^{B}$. Agent $A$ 's control variables are the probability $x$ of engaging in thinking ahead before investing and the probability $y$ of engaging in thinking on the spot in state $\theta_{2}$ before chosing an action. Therefore agent $A$ is looking for the solution to the constrained maximization program:

$$
\begin{aligned}
M P_{A} \equiv & \max _{x, y} x \widehat{\Lambda}\left[-I+\frac{\delta \pi}{2}+\frac{\delta \rho_{A}^{*}}{2}\right] \\
& +(1-x)\left[-I+\frac{\delta \pi}{2}+y \widehat{\Lambda}\left(\frac{\delta \rho_{A}^{*}}{2}\right)+(1-y) \frac{\delta \rho_{A}}{2}\right]
\end{aligned}
$$

subject to:

$$
\begin{gathered}
\widehat{\Lambda}\left[-I+\frac{\delta \pi}{2}+\frac{\delta \rho_{B}^{*}}{2}\right] \leq \\
x \widehat{\Lambda}\left[-I+\frac{\delta \pi}{2}+\frac{\delta \rho_{B}^{*}}{2}\right]+(1-x)\left[-I+\frac{\delta \pi}{2}+y \widehat{\Lambda}\left(\frac{\delta \rho_{B}^{*}}{2}\right)+(1-y) \frac{\delta \rho_{B}}{2}\right] .
\end{gathered}
$$

Other contracts that involve for instance choosing the safe action before learning whether it is optimal or, choosing the sub-optimal action once agents have learned which action is best are dominated for both agents and cannot therefore maximize $A$ 's payoff under the constraint that $B$ obtains at least $U_{\text {min }}^{B}$.

\footnotetext{
${ }^{14}$ An equivalent contract is to draw contract $C_{\alpha}$ with probability $y$ and to take the risky action in state $\theta_{2}$ with probability $(1-y)$.
} 
Forming the Lagrangian, and taking its partial derivatives with respect to $x$ and $y$ we obtain that:

$$
\frac{\partial L}{\partial x}(1-x)=(1-y) \frac{\partial L}{\partial y}-(1-x)(1+\vartheta)(1-\widehat{\Lambda})\left(-I+\frac{\delta \pi}{2}\right)
$$

where $\frac{\partial L}{\partial x}$ (resp. $\frac{\partial L}{\partial y}$ ) is the partial derivative of the Lagrange function with respect to $x$ (resp. $y$ ) and $\vartheta$ is the Lagrange multiplier of the constraint.

From the last inequality it is apparent that the solution to this program is $x^{*}=0$ and $y^{*} \in(0,1)$ if and only if:

$$
-I+\frac{\delta \pi}{2}+\widehat{\Lambda}\left(\frac{\delta \rho_{B}^{*}}{2}\right)>\widehat{\Lambda}\left[-I+\frac{\delta \pi}{2}+\frac{\delta \rho_{B}^{*}}{2}\right],
$$

which is true under assumptions A2 to A4. This establishes that the most efficient way for $A$ to deviate from his preferred course of action is to invest right away, to choose the risky action in state $\theta_{2}$ with probability $\left(1-y^{*}\right)$ and to think on the spot with probability $y^{*}$. This action-plan is implemented by offering party $B$ control with probability $y^{*}$, as party $B$ would then want to think on the spot in state $\theta_{2}$. Finally, the exact value of $y^{*}$ is given by:

$$
\begin{aligned}
-I+\frac{\delta \pi}{2}+ & y^{*} \widehat{\Lambda}\left(\frac{\delta \rho_{B}^{*}}{2}\right)+\left(1-y^{*}\right) \frac{\delta \rho_{B}}{2}= \\
\widehat{\Lambda} & {\left[-I+\frac{\delta \pi}{2}+\frac{\delta \rho_{B}^{*}}{2}\right] }
\end{aligned}
$$

To summarize, the following strategies support this subgame-perfect equilibrium:

- Equilibrium strategy for $A$ : at date 0, offer a stochastic contract committing to immediate investment and that implements $C_{R}$ with probability $1-y^{*}$ and $C_{B}$ with probability $y^{*}$. If the contract is accepted, invest at date 0 and if state $\theta_{2}$ is realized and $A$ has control, implement decision $r$. If $B$ has control, think and credibly reveal any new information to $B$.

If the offer is rejected, think and again credibly reveal any new information to $B$. If $A$ uncovers the optimal decision in state $\theta_{2}$ reveal it to $B$ and offer the first-best optimal complete contract to $B$ (either $C_{R}$ or $C_{S}$ depending on whether $A$ uncovers that $r$ or $s$ is optimal). Similarly, if $B$ reveals the optimal decision in state $\theta_{2}$ offer the first-best complete contract to $B$.

If $A$ learns nothing during that second sub-period of period 0 (from his own thinking or from $B$ ) repeat at date 1 the same strategy as at date 0 and continue doing so until investment takes place. ${ }^{15}$

\footnotetext{
${ }^{15}$ Note that nothing is changed if party $A$ offers initially $C_{A}$ instead of $C_{r}$, or $C_{\alpha}$ instead of $C_{B}$.
} 
- Equilibrium strategy for $B$ : at date 0, accept all contract offers with immediate investment that take support in $\mathbb{C} \backslash\left\{C_{S}\right\}$, provided that those offers put a weight of at least $y^{*}$ on the choice of $C_{B}$. In state $\theta_{2}$, when $B$ has control think on the spot and implement the optimal decision. Following a rejection at date 0, think in the second sub-period of date 0 and reveal any information to $A$. Then accept all first-best complete contract offers. Similarly, if $A$ reveals that decision $s$ (resp. $r$ ) is optimal in state $\theta_{2}$, accept all first-best complete contract offers. If neither party learns anything, repeat at date 1 the same strategy as at date 0 and continue doing so until a contract is accepted.

Proof of Corollary 1: immediate from previous results and noticing that now necessarily under $\boldsymbol{A} \mathscr{2}$ to $\boldsymbol{A} \mathbf{4}$,

$$
-I+\frac{\delta \pi}{2}+\frac{\delta \widehat{\Lambda} \rho_{A}^{*}}{2}>\widehat{\Lambda}\left[-I+\frac{\delta \pi}{2}+\frac{\delta \rho_{A}^{*}}{2}\right]=U_{\min }^{A} .
$$

Therefore, the proposer $B$ must obtain her most preferred path of action, i.e. $C_{B}$.

Proof of Proposition 4: Note that under assumption A6.b and A6.c, $U_{\min }^{B}=\widehat{\Lambda} U^{R F I}$ is the highest attainable payoff for $B$. From claim 2 above, this must be her equilibrium payoff. Also, under assumption A6.a thinking ahead of investing is costly for $A$ and is not his most preferred strategy.

Lemma 2: Under Assumptions A7 and A8, when the agents are uninformed in state $\theta_{2}$ under contract contract $C_{A}$ the only equilibrium that exists is a mixed strategy equilibrium, where both $A$ and $B$ randomize between stopping and not stopping at any time $\tau \geq \widehat{\tau}+1$.

Proof of Lemma 2: We begin by showing that a pure strategy equilibrium cannot exist. To see this, note that withholding all information (whether $R_{k}=\underline{R}_{k}$ or $R_{k}=\bar{R}_{k}$ ) from time $\tau$ onwards is a best response for $B$ whenever:

$$
\left[\delta\left(1-\lambda_{A}\right)\right]^{\left(\tau_{A}-\tau\right)} \bar{R}_{B} \geq S_{B}\left(1-\sum_{t=1}^{\left(\tau_{A}-\tau\right)}\left(\lambda_{A}\left(1+\left(1-\lambda_{A}\right)^{t} \delta^{t}\right)\right),\right.
$$

where we define $A$ 's stopping time $\tau_{A}$ under the assumption that $B$ always discloses $\bar{R}_{k}$ up to time $\tau_{A}$.

But if $B$ stops disclosing $\bar{R}_{k}$ at some earlier time $\tau$, $A$ 's optimal stopping time in turn may change, as $A$ can then no longer update his beliefs after time $\tau$. Let $\tau_{B}$ and $\nu_{\tau_{B}}$ respectively 
denote the time when $B$ stops disclosing $\bar{R}_{k}$ and $A$ 's belief at time $\tau_{B}$. Also, let

$$
\widehat{\lambda}_{A}=\frac{\lambda_{A}}{1-\left(1-\lambda_{A}\right) \delta}
$$

then we have either:

$$
\nu_{\tau_{B}} \bar{R}_{A}+\left(1-\nu_{\tau_{B}}\right) \underline{R}_{A} \geq \widehat{\lambda}_{A}\left(\nu_{\tau_{B}} S_{A}+\left(1-\nu_{\tau_{B}}\right) \underline{R}_{A}\right)
$$

or

$$
\nu_{\tau_{B}} \bar{R}_{A}+\left(1-\nu_{\tau_{B}}\right) \underline{R}_{A}<\widehat{\lambda}_{A}\left(\nu_{\tau_{B}} S_{A}+\left(1-\nu_{\tau_{B}}\right) \underline{R}_{A}\right) .
$$

That is, it is best for $A$ to either stop thinking at time $\tau_{B}$, or to continue thinking until he learns the optimal action.

In the latter situation we have $\tau_{A} \longmapsto \infty$ so that (8) no longer holds and $B$ 's best response is to disclose $\bar{R}_{k}$ at time $\tau_{B}$. Similarly, in the former situation, we have $\tau_{A}=\tau_{B}$, but if this is anticipated $B$ 's best response is to stop disclosing $\bar{R}_{k}$ even earlier. In sum, whether (10) holds or not, a pure strategy equilibrium in stopping times does not exist.

There exists a mixed strategy equilibrium, as we now establish, where both $A$ and $B$ randomize between stopping and not stopping at any time $\tau \geq \widehat{\tau}+1$. Time $\widehat{\tau}$ is the first time when $\nu_{\widehat{\tau}}$ is such that

$$
\nu_{\widehat{\tau}-1} \bar{R}_{A}+\left(1-\nu_{\widehat{\tau}-1}\right) \underline{R}_{A}<\widehat{\lambda}_{A}\left(\nu_{\widehat{\tau}-1} S_{A}+\left(1-\nu_{\widehat{\tau}-1}\right) \underline{R}_{A}\right)
$$

but

$$
\nu_{\widehat{\tau}} \bar{R}_{A}+\left(1-\nu_{\widehat{\tau}}\right) \underline{R}_{A} \geq \widehat{\lambda}_{A}\left(\nu_{\widehat{\tau}} S_{A}+\left(1-\nu_{\widehat{\tau}}\right) \underline{R}_{A}\right) .
$$

If we denote by $\phi$ the probability that $A$ stops thinking at any time $\tau \geq \widehat{\tau}$ and by $\varphi_{\tau-1}$ the probability that $B$ stops disclosing $\bar{R}_{k}$ at $\tau-1$ then for any $\tau$ the following two equations must hold in equilibrium. For $A$ we must have:

$$
\begin{aligned}
\rho^{A}\left(\nu_{\tau-1}\right)= & \delta\left\{\nu_{\tau-1} S_{A}\left(\varphi_{\tau-1} \lambda_{A}+\left(1-\varphi_{\tau-1}\right) \Lambda\right)+\left(1-\nu_{\tau-1}\right) \lambda_{A} \underline{R}_{A}+\right. \\
& {\left.\left[\nu_{\tau-1}\left(\left(1-\varphi_{\tau-1}\right)(1-\Lambda)+\varphi_{\tau-1}\left(1-\lambda_{A}\right)\right)+\left(1-\nu_{\tau-1}\right)\left(1-\lambda_{A}\right)\right] \rho^{A}\left(\nu_{\tau}\right)\right\}, }
\end{aligned}
$$

where

$$
\rho^{A}\left(\nu_{\tau}\right)=\nu_{\tau} \bar{R}_{A}+\left(1-\nu_{\tau}\right) \underline{R}_{A},
$$

and

$$
1-\nu_{\tau}=\varphi_{\tau-1}\left(1-\nu_{\tau}\right)+\frac{\left(1-\varphi_{\tau-1}\right)\left(1-\nu_{\tau-1}\right)}{\nu_{\tau-1}\left(1-\lambda_{B}\right)+\left(1-\nu_{\tau-1}\right)}
$$


Similarly, for party $B$ we must have:

$$
S_{B}=\lambda_{A} S_{B}+\left(1-\lambda_{A}\right)\left[\phi \bar{R}_{B}+(1-\phi) \delta S_{B}\right]
$$

or,

$$
\phi=\frac{S_{B}(1-\delta)}{\bar{R}_{B}-\delta S_{B}}
$$

In other words, for any $\tau, B$ must be indifferent between stopping disclosing $\bar{R}_{k}$ at $\tau+1$ or at $\tau$. If $B$ stops at $\tau+1$ (and therefore discloses $\bar{R}_{k}$ in period $\tau$ ) she gets $S_{B}$ at $\tau$. If she does not disclose $\bar{R}_{k}$ at $\tau$, then $A$ discovers $\bar{R}_{k}$ at $\tau$ with probability $\lambda_{A}$, in which case $B$ gets again $S_{B}$. With probability $\left(1-\lambda_{A}\right), A$ does not discover $\bar{R}_{k}$ and stops with probability $\phi$, in which case $B$ gets $\bar{R}_{B}$; and finally with probability $(1-\phi)$ party $A$ continues learning, in which case $B$ 's continuation value is $\delta S_{B}$ (as $B$ discloses $\bar{R}_{k}$ at $\tau+1$ ).

As can be readily checked, these equations admit a unique solution $\phi \in[0,1]$ and $\varphi_{\tau-1} \in$ $[0,1]$ under Assumptions A7 and A8.

Proposition 5: Under Assumptions A1, A4, A7, $\boldsymbol{A 8}$ and $\boldsymbol{A 9}$ agent $A$ (weakly) prefers the coarse contract $C_{R}$ at date 0 to contracts $C_{A}, C_{B}$ or $C_{A B}$, and therefore there is an equilibrium in which no incomplete contract is proposed and accepted.

Proof of Proposition 5: Under assumption A8 and A9 $C_{R}$ is (weakly) preferred by both agents to $C_{A}$. In addition under assumptions $\mathbf{A} \mathbf{1}$ and $\mathbf{A 7}, C_{R}$ is also preferred by $A$ to $C_{S}$ which is again preferred by $A$ to $C_{B}$ as $S_{A}>V_{B}^{A}$, where $V_{B}^{A}$ is $A$ 's payoff under contract $C_{B}$ :

$$
\begin{aligned}
V_{B}^{A} \equiv & \sum_{\tau=1}^{\tau_{B}}\left[\nu \bar{R}_{A} \lambda_{B}\left(1+\left(1-\lambda_{B}\right)^{\tau} \delta^{\tau}\right)+(1-\nu) S_{A} \Lambda\left(1+(1-\Lambda)^{\tau} \delta^{\tau}\right)\right]+ \\
& \delta^{\tau_{B}}\left(\nu_{\tau_{B}} \bar{R}_{A}+\left(1-\nu_{\tau_{B}}\right) \underline{R}_{A}\right),
\end{aligned}
$$

and where $\tau_{B}$ is similarly defined as $\widehat{\tau}$ but with $A$ 's and $B$ 's roles interchanged.

Also, $C_{R}$ is (weakly) preferred by both agents to $C_{A B}$. Finally, by Claim 2 in the Appendix, $C_{B}$ will not be offered in equilibrium even when $B$ prefers $C_{B}$ to $C_{R}$.

Proposition 6: Under Assumptions A1, A4, A7, A8, A9 and A10, a cutoff $\bar{I}<\frac{\delta \pi}{2}$ exists such in equilibrium: If $I \geq \bar{I}$ agents think ahead before commiting to invest in the venture; Otherwise, $A$ and $B$ sign the coarse contract $C_{R}$ at date 0 . 
Proof of Proposition 6: The cutoff $\bar{I}$ is defined by equating $A$ 's expected payoffs under the two contracting strategies. By offering the coarse contract $C_{R}$ at date 0 party $A$ gets:

$$
-I+\frac{\delta \pi}{2}+\frac{\delta \rho_{A}}{2}
$$

And by first thinking ahead and offering a complete contingent contract $A$ gets:

$$
\widehat{\Lambda}\left[-I+\frac{\delta \pi}{2}+\frac{\delta \rho_{A}^{*}}{2}\right],
$$

given that Assumption A9 holds. The cutoff $\bar{I}$ is then defined by:

$$
\bar{I}=\frac{\delta \pi}{2}-\frac{\delta}{2}\left(\frac{\widehat{\Lambda} \rho_{A}^{*}-\rho_{A}}{1-\widehat{\Lambda}}\right)<\frac{\delta \pi}{2} .
$$

To see that an offer of $C_{R}$ at date 0 -which is accepted by $B$-is an equilibrium when $I<\bar{I}$, note first that under Assumptions $\boldsymbol{A}$ 7, $\boldsymbol{A} \boldsymbol{8}$ and $\mathbf{A 9}$, an offer of $C_{R}$ at date 0 provides a higher payoff than $C_{A}, C_{B}$ or $C_{A B}$ to both $A$ and $B$ (as established in Proposition 5). Moreover, when $I \leq \bar{I}$, both agents are also better off signing $C_{R}$ at date 0 than thinking ahead. Therefore, $B$ will accept an offer of $C_{R}$ at date 0 and $A$ will indeed offer $C_{R}$.

Finally, when $I \in\left[\bar{I}, \frac{\delta \pi}{2}\right), A$ is better off delaying a contract offer and thinking ahead if $B$ also thinks and shares her thoughts. Similarly, $B$ 's best response to $A$ thinking ahead is to also think ahead and share her thoughts.

Proposition 7: Under Assumptions A1, A3, A7, A8, A9 and A11, an offer $C_{R}$ from $A$ at $t=0$ is an equilibrium of the contracting game when

$$
x^{*}=\frac{1}{\underline{R}_{B}-S_{B}}\left(\frac{2 I}{\delta}-\pi-S_{B}\right)
$$

is close enough to zero.

Proof of Proposition 7: Given their prior beliefs both agents prefer the risky to the safe action under assumption A1. Agent $A$ can make an offer $C_{R}$ at $t=0$ and have $B$ accept it when it is common knowledge that none of the players are informed.

We shall assume that $B$ has pessimistic out-of-equilibrium beliefs and that if a contract is not immediately accepted at time $t=0, B$ believes that $A$ knows that $R_{k}=\underline{R}_{k}$ when he makes an offer $C_{R}$ and therefore $B$ rejects any offer $C_{R}$ past period 0 given Assumption A11.

Thus after $t=0, A$ can no longer get an offer $C_{R}$ accepted by $B$. Under Assumption A11, the only offers $B$ will accept after time $t=0$ are $C_{S}$ and contract $C_{x^{*}}$, where $r$ is 
chosen with probability $x^{*}$ defined in equation (6) and $s$ is chosen with probability $\left(1-x^{*}\right)$ when $R_{k}=\underline{R}_{k}$. Therefore, $A$ 's payoff when thinking ahead of investing is at most

$$
\widehat{\Lambda}\left[-I+\frac{\delta \pi}{2}+\frac{\delta}{2}\left(\nu S_{A}+(1-\nu)\left(x^{*} \underline{R}_{A}+\left(1-x^{*}\right) S_{A}\right)\right)\right]
$$

which is dominated by $A$ 's payoff under contract offer $C_{R}$ at time $t=0$ :

$$
-I+\frac{\delta \pi}{2}+\frac{\delta}{2}\left(\nu \bar{R}_{A}+(1-\nu) \underline{R}_{A}\right),
$$

when $x^{*}$ is low enough, by Assumption A1.

Moreover, under Assumption A8 and A9 $A$ weakly prefers contract $C_{R}$ to $C_{A}$.

Proposition 8: Under the same Assumptions as in Proposition 7 and Assumptions A12 and $\boldsymbol{A} \mathbf{1 3}$, and provided that

$$
x^{*}=\frac{1}{\underline{R}_{B}-S_{B}}\left(\frac{2 I}{\delta}-\pi-S_{B}\right)
$$

is sufficiently close to 1, the unique subgame-perfect equilibrium is such that:

i) A offers a preliminary contract to $B$ at $t=0$ with the following terms: a) the agents commit to invest once they have thought through $\left.R_{k} ; b\right)$ if $R_{k}=\underline{R}_{k}$ then action $r$ is chosen in state $\theta_{2}$; c) if $R_{k}=\bar{R}_{k}$ then action $s$ is chosen with probability $\chi$ and action $r$ with probability $(1-\chi)$ in state $\theta_{2}$, where $\chi$ is given by:

$$
\left.-I+\frac{\delta \pi}{2}+\frac{\delta}{2}\left[\nu\left(\chi S_{B}+(1-\chi) \bar{R}_{B}\right)+(1-\nu) \underline{R}_{B}\right)\right]=0
$$

ii) both agents think ahead and share their thoughts.

Proof of Proposition 8: Note first that if (11) holds the preliminary contract is acceptable to $B$. Second, under Assumption A12, $A$ strictly prefers the preliminary contract to thinking ahead and settling on either contracts $C_{S}$ or $C_{x^{*}}$. To see this, consider, $A$ 's exante maximization problem with respect to $\chi$ and $x$ :

$$
\begin{aligned}
& \max _{\chi, x}\left\{-I+\frac{\delta \pi}{2}+\frac{\delta}{2}\left[\nu\left(\chi S_{A}+(1-\chi) \bar{R}_{A}\right)+\right.\right. \\
& \left.\left.(1-\nu)\left(x \underline{R}_{A}+(1-x) S_{A}\right)\right]\right\}
\end{aligned}
$$

subject to:

$$
\begin{gathered}
-I+\frac{\delta \pi}{2}+\frac{\delta}{2}\left[\nu\left(\chi S_{B}+(1-\chi) \bar{R}_{B}\right)+\right. \\
\left.(1-\nu)\left(x \underline{R}_{B}+(1-x) S_{B}\right)\right]=0 \\
42
\end{gathered}
$$


Substituting for $x$, this problem is equivalent to the unconstrained problem:

$$
\begin{aligned}
& \max _{\chi} \nu\left(\chi\left(S_{A}-\bar{R}_{A}\right)+\bar{R}_{A}\right)+(1-\nu) S_{A}+ \\
& \left(\underline{R}_{A}-S_{A}\right)\left[\frac{\frac{2 I}{\delta}-\pi-(1-\nu) S_{B}-\nu\left(\chi\left(S_{B}-\bar{R}_{B}\right)+\bar{R}_{B}\right)}{\underline{R}_{B}-S_{B}}\right]
\end{aligned}
$$

Differentiating with respect to $\chi$ we observe that the coefficient with respect to $\chi$ is strictly positive under Assumption A12, which means that $A$ would like to set $\chi$ as high as possible and $x$ as low as possible. The best contract for $A$ is then obtained by setting $x=0$.

Third, $A$ and $B$ prefer the preliminary contract to $C_{R}$ under Assumption A3 given that $x^{*}$ is close to 1 , as they then already prefer to think ahead and settle on contracts $C_{S}$ and $C_{x^{*}}$ to signing $C_{R}$.

Fourth, A's continuation best-response following acceptance of contract $C_{\alpha}$ is to think ahead, for no investment can take place unless $A$ reveals the value of $R_{k}$.

Fifth, $A$ is clearly better off disclosing $\underline{R}_{k}$ under Assumptions $\mathbf{A} \mathbf{1}$ and $\mathbf{A 7}$. He is also better off disclosing $\bar{R}_{k}$ under Assumption A13.

Finally, under Assumptions $\mathbf{A 8}$ and $\mathbf{A 9}$ the agents weakly prefer $C_{R}$ to $C_{A}$, but $C_{R}$ in turn is dominated by $C_{\alpha}$ when $x^{*}$ is close to 1 .

Lemma 4 (The Congruence Principle): it is weakly optimal for the contracting agents to begin by signing a preliminary agreement which establishes how the agents will share the profits from the venture.

Proof of Lemma 4: Let $\kappa_{l}^{j} \in\left\{\underline{R}_{k}, \bar{R}_{k}, \varnothing\right\}$ denote the payoffs communicated by agent $j=A, B$ to the other agent up to time $l$, and let $I\left(\kappa_{t}^{j}\right)$ denote an investment plan specifying a (possibly random) time $t$ contingent on the payoffs communicated by the agents up to time $t$, at which investment is sunk. Also, let $\left\{a\left(\theta, \kappa_{\tau(\theta)}^{j}\right)\right\}$ denote a plan to take action $a\left(\theta, \kappa_{\tau(\theta)}^{j}\right)$ in state $\theta$ at (possibly random) time $\tau(\theta)$ contingent on the payoffs communicated by the agents up to time $\tau(\theta) \geq t+1$.

Then $\rho\left(a\left(\theta_{2}, \kappa_{\tau}^{j}\right)\right)$ denotes the expected revenue obtained in state $\theta_{2}$ under investment plan $I\left(\kappa_{t}^{j}\right)$ and action plan $\left\{a\left(\theta, \kappa_{\tau(\theta)}^{j}\right)\right\}$, and $V$ denotes the maximum value of the venture at date 0 :

$$
V=E\left\{\max _{I\left(\kappa_{t}^{j}\right) ; a\left(\theta, \kappa_{\tau(\theta)}^{j}\right)} \delta^{\tau(\theta)} \rho\left(a\left(\theta, \kappa_{\tau(\theta)}^{j}\right)\right)-\delta^{t} I\left(\kappa_{t}^{j}\right)\right\}
$$


We shall argue that by offering a preliminary contract such that the agents share profits, with $\alpha_{k} \geq 0$ denoting the share of profits of party $k=A, B$, the proposer can achieve the highest feasible payoff $V-U_{\min }^{R}$.

Under such a contract each party's payoff for any given subsequent investment plan $I\left(\kappa_{t}^{j}\right)$ and action plan $\left\{a\left(\theta, \kappa_{\tau(\theta)}^{j}\right)\right\}$ is:

$$
\alpha_{k} E\left\{\delta^{\tau(\theta)} \rho\left(a\left(\theta, \kappa_{\tau(\theta)}^{j}\right)\right)-\delta^{t} I\left(\kappa_{t}^{j}\right)\right\}
$$

Thus both agents have aligned objectives on the choice of investment and action plan given any $\alpha_{k} \geq 0$ and will agree on a plan that achieves $V$. It then suffices for the proposer to choose $\alpha_{B}$ such that

$$
\alpha_{B} E\left\{\delta^{\tau(\theta)} \rho\left(a\left(\theta, \kappa_{\tau(\theta)}^{j}\right)\right)-\delta^{t} I\left(\kappa_{t}^{j}\right)\right\}=U_{\min }^{R}
$$

